\title{
Masked stereolithography of hydroxyapatite bioceramic scaffolds through an integrative approach: From powder tailoring to evaluation of 3D printed parts properties
}

\author{
P. Navarrete-Segado, ${ }^{\text {a,b }}$ M. Tourbin, ${ }^{\mathrm{b}}$ C. Frances, ${ }^{\mathrm{b}}$ D. Grossin ${ }^{\mathrm{a}, *}$

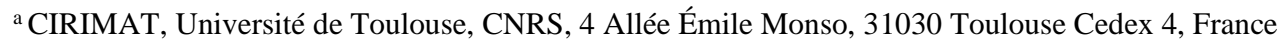 \\ ${ }^{\text {b }}$ Laboratoire de Génie Chimique, Université de Toulouse, CNRS, 4 Allée Émile Monso, 31432 Toulouse Cedex 4, France
}

\begin{abstract}
In this paper, the tailoring of hydroxyapatite powders properties for the preparation of highly hydroxyapatite-loaded photocurable organic slurries was discussed. A methodical study was conducted to investigate suspensions properties and processability to find the most outstanding formulation for the production of hydroxyapatite scaffolds by stereolithography-based additive manufacturing technique. A debinding-sintering process was designed to avoid the formation of cracks during the pyrolysis of the resin and sintering of the ceramic part. A total porosity of $35 \mathrm{vol} . \%$ was observed for the scaffolds with an interconnected macroporosity, which could facilitate the flow transport of nutrients necessary to maintain living cells. A compression strength of $4.9 \pm 0.3 \mathrm{MPa}$ was obtained for the specimens printed diagonally ( $45^{\circ}$ to the printing stage surface). A slow degradation rate was shown for the printed parts mainly due to the high degree of crystallinity and the intrinsic stability of the hydroxyapatite phase. Our findings indicate that the tailoring of hydroxyapatite powders is needed for better processability as filler in photocurable suspensions. Moreover, it was demonstrated the feasibility of printing hydroxyapatite parts showing promising results for their application in surgery in the case of minor or non-load bearing implants requiring slow resorption properties.
\end{abstract}

Keywords: hydroxyapatite, rheology, sedimentation, suspensions, mechanical properties, VAT photopolymerisation; additive manufacturing

*Corresponding author: david.grossin@ensiacet.fr 


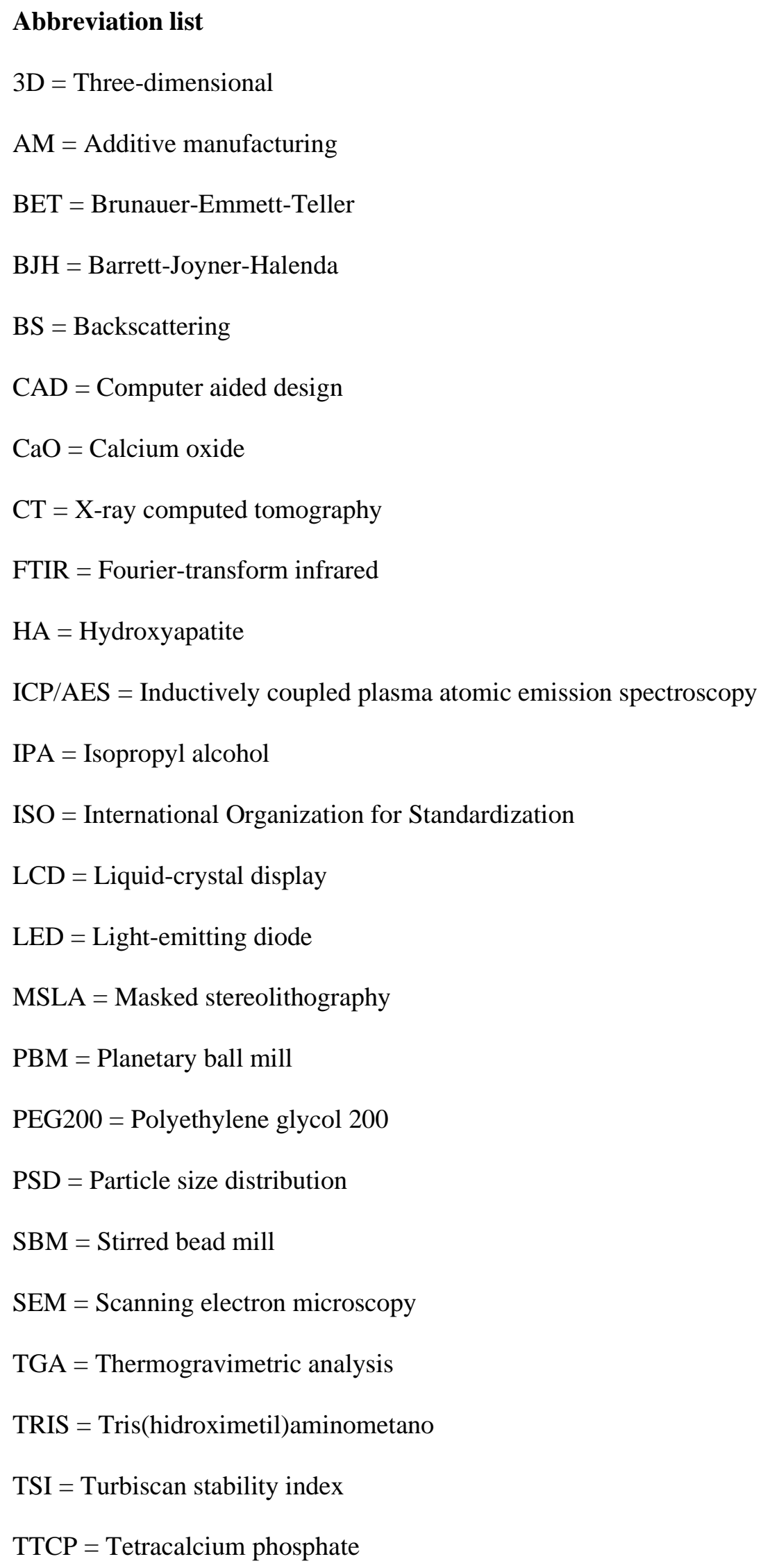


$\mathrm{UV}=$ Ultraviolet

$\mathrm{XRD}=\mathrm{X}$-ray diffraction

$\alpha$-TCP $=\alpha$-Tricalcium phosphate

$\beta$-TCP $=\beta$-Tricalcium phosphate

\section{Symbols}

$\mathrm{x}_{10}=$ the $10^{\text {th }}$ percentile $[\mu \mathrm{m}]$ (diameter of a sphere at which $10 \%$ of the particles in the sample are smaller) $\mathrm{x}_{50}=$ Median particle size $[\mu \mathrm{m}]=$ the $50^{\text {th }}$ percentile (diameter of a sphere at which $50 \%$ of the particles in the sample are smaller)

$\mathrm{X}_{90}=$ The $90^{\text {th }}$ percentile $[\mu \mathrm{m}]$ (diameter of a sphere at which $90 \%$ of the particles in the sample are smaller) 


\section{Introduction}

People are living longer than ever before with the median age projected to increase by 4.5 years between 2019 and 2050 [1]. This ageing increase leads to a growth of incidence of bone injuries and deficiencies [2]. In the last decades, the rising interest for new efficient therapies has brought wide progress on the use of biomaterials such as calcium phosphate-based bioceramics [3]. Reparation of fractured or diseased bone needs to start from a scaffold or three-dimensional (3D) construct providing the necessary support for the attachment, growth, and proliferation of bone cells [4]. Hydroxyapatite (HA) is one of the most commonly used calcium phosphates bioceramic phases for bone tissue engineering in orthopaedic and dental communities for its excellent biocompatibility, slow degradation, and osteointegration [5]. HA can withstand resorption during healing providing a good scaffold for natural bone growth, their slow resorption properties could be an advantage in maintaining the volumetric stability of the augmented bone. The presence of hard tissue around a dental implant is crucial for the long-term success of implant placement in surgical techniques such as guided bone regeneration and autogenous bone blocks transfer for dental implant placement [6-8]. For block grafts used in regeneration, an ideal biomaterial, should meet at least the following characteristics: (i) biocompatibility and controlled biodegradability with sufficient durability to carry out the task for which it was developed [9], (ii) a porous and interconnected design enabling osteoblasts $(15-50 \mu \mathrm{m})$ and stem cells $(5-12 \mu \mathrm{m})$ migration, proliferation, and flow transport of nutrients and metabolic waste through the pores [10] (ideal size of the micropores should be at least $400 \mu \mathrm{m}$ ) [11], (iii) mechanical properties matching those of the target tissue at the site of implantation [12], (iv) suitable surface chemistry for cell anchorage, promotion and differentiation [12].

Additive manufacturing (AM) technologies have emerged as an excellent method to fabricate parts suitable for their use in bone tissue engineering applications [13]. They allow the production of allograft custom blocks with defined and reproducible internal structures [14]. Depending on the AM technique, the process basis, methodology, and primary feedstock properties can vary widely $[15,16]$. Techniques included in the VAT photopolymerization category uses a laser or UV-light source to cross-link a 
photocurable resin feedstock (composed of a photoinitiator, monomers, or oligomers, reactive diluents, and various additives such as bioceramic powders) [17].

Masked stereolithography (MSLA), also called LCD technique, is the last of these technologies arriving on the market and it has substantially grown in the last years [18]. It uses a direct and perpendicular UV light projection from an array of LEDs and an LCD device acting as a mask to selectively polymerize the photocurable feedstock. Some advantages of this LCD technique are the low cost compared with other technologies (laser SLA, DLP...) and the good resolution, although they have a shorter service life and need to be replaced regularly [19]. This and similar techniques have been recently employed for the production of calcium phosphates-based parts [17,20,21]. However, still, some research is needed on the intrinsic relationship between ceramic powder characteristics, solid loading, and dispersion behaviour on the ceramic-loaded photocurable slurry properties [22]. Dispersed powder feedstock inducing a low increase of viscosity, keeping the stability of the suspensions, and showing a good sintering behaviour are pursued since these are crucial properties for a correct performance during the printing process as well as during post-treatments [23-25]. The maximum viscosity for a slurry intended for VAT photopolymerization is approximately 5 Pa.s at a low shear rate depending on the printer design [26]. Then, certain stability of the suspensions should be reached avoiding the sedimentation of the particles, which could block the LCD screen and UV light during the printing process [23]. It is also important to use particles which size allows the subsequent debinding-sintering process preventing the formation of cracks. More often, this is achieved by calcium phosphate particles within the micron range $(1-20 \mu \mathrm{m})$ [27]. This can be reached by modifying the powder particle size distribution through different processes (e.g., milling, sieving...).

Thus, the objective of the present study is to evaluate the effect of the HA-tailored powders with different characteristics on the properties of the highly HA-loaded photocurable slurries. First, the powder showing the most outstanding performance in terms of slurry viscosity $\left(<5\right.$ Pa.s at a low shear rate $\left.\left(1 \mathrm{~s}^{-1}\right)\right)$, and dispersion stability (Turbiscan stability index < 2) during the whole process will be found through comparison. Then, the processability of the suspension will be tested with the printing of HA parts with 
controlled macroporosity (pore size $>400 \mu \mathrm{m}$ ). Finally, the mechanical properties and the dissolution rate of the scaffolds will be determined to assess the HA bioceramic scaffolds efficacy as a bone substitute.

\section{Material and methods}

\subsection{Materials}

\subsubsection{Stoichiometric hydroxyapatite powders}

Stoichiometric hydroxyapatite powder $\left.\left(\mathrm{HA}, \mathrm{Ca}_{10}\left(\mathrm{PO}_{4}\right)_{6}(\mathrm{OH})_{2}\right)\right)$ was used as filler for the suspensions prepared in the present work. The initial powder was produced by Urodelia (SA Company, Saint-Lys, France) via a wet precipitation process (product reference 206.93.003). A deeper explanation of the production of this powder through wet-chemical precipitation was offered in a previous work [28].

HA-filled organic slurries for the MSLA process were produced with three kinds of HA powders (two of them produced by different milling processes) for suspension properties comparison (e.g., stability, rheology...):

- HA $_{\text {Initial }}$ powder: the starting HA powder was used as received.

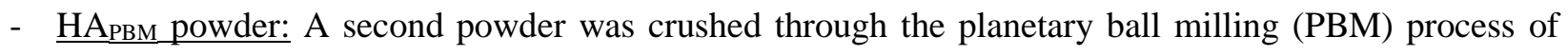
$\mathrm{HA}_{\text {Initial. }}$ A Retsch ${ }^{\circledR}$ planetary-ball mill S 1000 was used to reduce the size of the largest agglomerates present in $\mathrm{HA}_{\text {Initial }}$ powder. A previously weighted amount of powder $(140 \mathrm{~g})$ is poured inside of the 250 $\mathrm{ml}$ alumina grinding jar with lid together with the following alumina grinding balls (bulk density: 3600 $\mathrm{kg} / \mathrm{m}^{3}$, mass: $346 \mathrm{~g}$ ): 3 balls of $30 \mathrm{~mm}+12$ balls of $20 \mathrm{~mm}+9$ balls of $10 \mathrm{~mm}$. Then, a ball filling degree of 0.38 (to total chamber volume) and powder filling degree of 0.44 (to ball-filled chamber volume) were used. After one hour of the milling process at $500 \mathrm{rpm}$, the HAРBM powder was recovered.

- $\underline{H A}_{\text {sBM powder: }}$ Stirred bead milling process (SBM) was performed in a commercial equipment Labstar manufactured by NETZSCH (Germany) to reduce the size of the HA particles present in $\mathrm{HA}_{\text {Initial }}$ powder. The detailed scheme of this SBM Labstar equipment and the experimental procedure were already detailed in previous works $[29,30]$. A water volume was added to the dispersing tank followed by the 
gradual addition of the weighted HA powder amount to have a final suspension batch of $2 \mathrm{~kg}$ with a 10 wt. $\%$ of solid concentration. It was stirred during 10 minutes at $600 \mathrm{rpm}$ at $25^{\circ} \mathrm{C}$ before starting the pumping and comminution in the milling chamber. Yttrium Stabilized Zirconia 95\% (YSZ, $\left.\mathrm{ZrO}_{2}\right)$ micro grinding beads (Zirmil ${ }^{\circledR}$ Y from WAB-Group) were used for the milling in a $85 \%$ of chamber filling degree (total volume chamber 0.481). The nominal size of the beads was $0.35-0.45 \mathrm{~mm}$ (laser diffraction particle analysed values $x_{10}=0.32 \pm 0.00 \mathrm{~mm}, x_{50}=0.39 \pm 0.00 \mathrm{~mm}$, and $x_{90}=0.45 \pm 0.00$, refractive and absorption indices of 2.148 and 1.000) with a density of $6020 \mathrm{~kg} \cdot \mathrm{m}^{-3}$ (bulk density: $3700 \mathrm{~kg} \cdot \mathrm{m}^{-3}$ ). The operational conditions were a stirrer speed of $7.7 \mathrm{~m} \cdot \mathrm{s}^{-1}(2000 \mathrm{rpm})$, a constant product flow rate 8.8 $\mathrm{x} 10^{-6} \mathrm{~m}^{3} \cdot \mathrm{s}^{-1}$, and specific energy of $720 \mathrm{~kJ} \cdot \mathrm{kg}^{-1}$ (it was equivalent to 60 minutes of the process). From now on, we will refer to this ground powder as $\mathrm{HA}_{\mathrm{SBM}}$. After this wet grinding process, the HA $\mathrm{A}_{\mathrm{SBM}}$ powder had to be vacuum filtered, washed, and dried at $70^{\circ} \mathrm{C}$ overnight before its utilization as filler.

\subsubsection{Photocurable resin components}

The commercial photoreactive resin Dentifix-3D, Modelling HR (high reactive) transparent (clear), from FunToDo ${ }^{\circledR}$ was used as a monomeric and photoinitiator base for the preparation of the organic suspensions. The exact composition of the resin is proprietary information; however, we could describe it as a mixture of approximately 50:50:1 in weight of acrylate monomers, glycol diacrylate monomers, and phosphine oxide base photoinitiator. To improve the rheological behaviour of the suspensions the addition of a reactive diluent was considered. Polyethylene glycol 200 (PEG200) was tested as a diluent and after a heuristic research process, it was found that the maximum amount that could be added without compromising the resin reactivity and performance was 25 vol. \%. This volume proportion 3:1 of resin base:diluent was kept constant for all the slurries prepared during the study.

\subsubsection{Preparation of HA-filled photocurable suspensions}

A series of HA-filled photocurable suspensions were prepared to contain different types $\left(\mathrm{HA}_{\text {Initial, }}\right.$ $\left.\mathrm{HA}_{\mathrm{PBM}}, \mathrm{HA}_{\mathrm{SBM}}\right)$ and concentrations of HA powders for comparison. The preparation consisted of the 
mixing/homogenization between the HA powder and the organic resin via the PBM process (same equipment used for the preparation of $\left.\mathrm{HA}_{\mathrm{PBM}}\right)$. It started with the addition of a previously weighted amount of HA powder (depending on the final concentration) into the grinding jar already containing the same proportion of grinding balls mentioned in the preparation of $\mathrm{HA}_{\mathrm{PBM}}$. Then, after pouring the organic resin (mass: 50g, already containing PEG200) the jar was closed with the lid and stirred by the PBM process at about $500 \mathrm{rpm}$ for $30 \mathrm{~min}$. Just after finishing the process, the resulting HA-filled suspensions were collected for rheology and dispersion stability evaluation and its utilization in an MSLA apparatus. A general view of the HA-filled photocurable slurry preparation is illustrated in Figure 1.

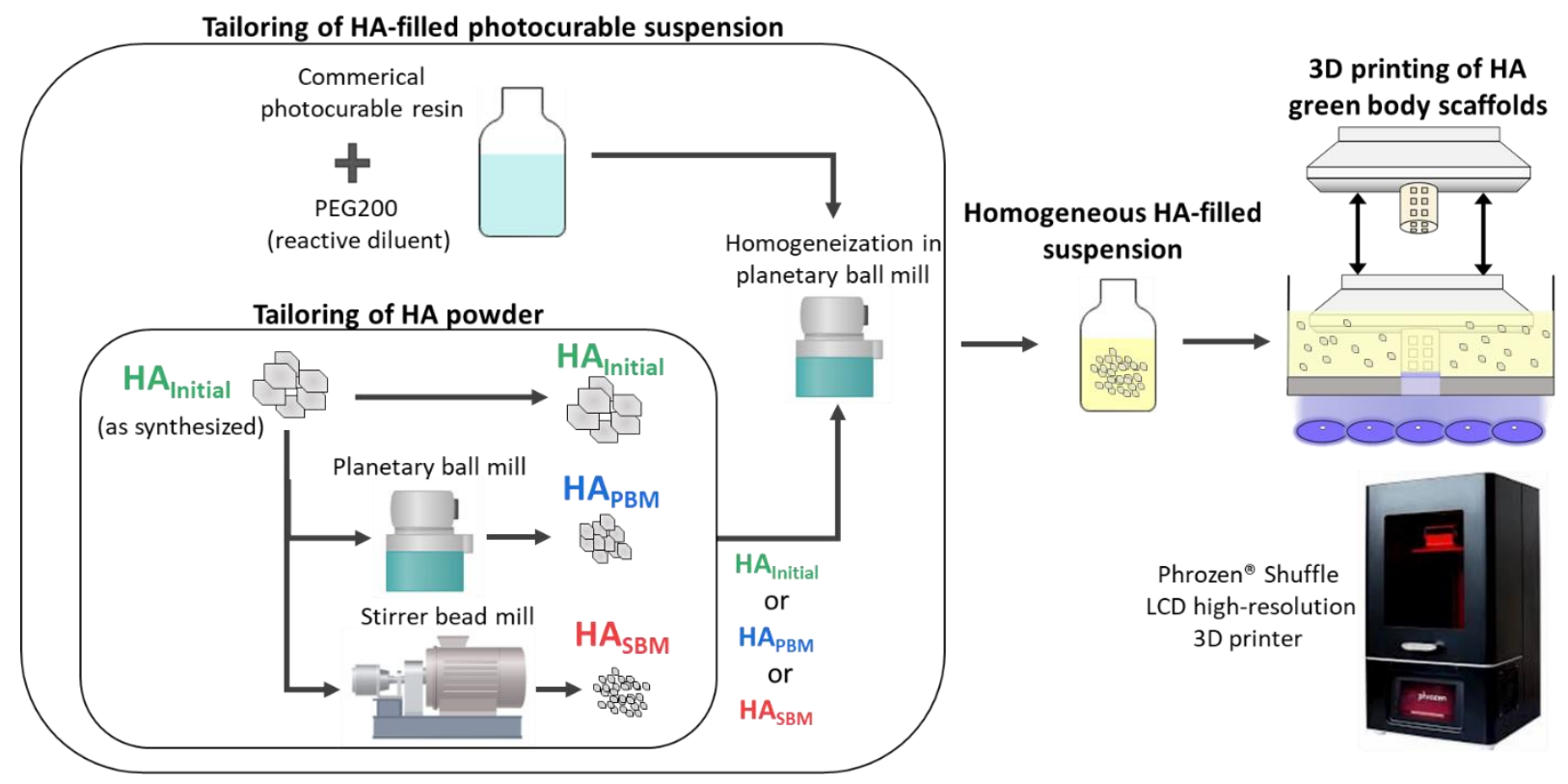

Figure 1. HA-filled photocurable slurry preparation process.

\subsection{Production of HA scaffolds}

\subsubsection{D printing of HA scaffolds via masked stereolithography}


Three-dimensional bioceramic green bodies (HA/organic resin composite) were printed by intermittent and selective cross-linking of HA-filled suspension layers. A commercial MSLA equipment, Phrozen ${ }^{\circledR}$ Shuffle, LCD high-resolution 3D-printer was used [31]. It uses an LCD screen with a 5.5-inch 2K resolution reaching an XY resolution of $47 \mu \mathrm{m}$. A ParaLED matrix 1.0 optical engine is used as a UV light source with a $90 \%$ optical uniformity (better than conventional COB LED). The produced UV light has a wavelength of $405 \mathrm{~nm}$ and a power of 50W.

The process started by loading the previously designed 3D model (Figure 2) into the printer software. Since compression strength analyses were planned for the samples, the 3D model was designed according to the requirements described in the standard ISO 13175-3:2012 for the tests. The scaffolds model design consisted of a cylinder with $10 \mathrm{~mm}$ of diameter and $15 \mathrm{~mm}$ of height, with controlled internal and interconnected macro-porosity having circular overtures of $1000 \mu \mathrm{m}$ in all three directions. Open-source FreeCAD and Chitubox basic software were employed for the design, creation of supports, placement of the models, and slicing. The resin profile was settled by fixing the printing parameters, which are described in Table 1. Basic information refers to the parameters followed during the whole process such as layer thickness, stage down speed, and lighting delay. Then, there is a distinction in the table between the printing parameters (cure time, stage lift height, and up speed) for the first 6 layers (burn layers) and the rest of the layers (normal layers). The same parameters profile was used for the printing of the scaffolds during the study. 
(a)

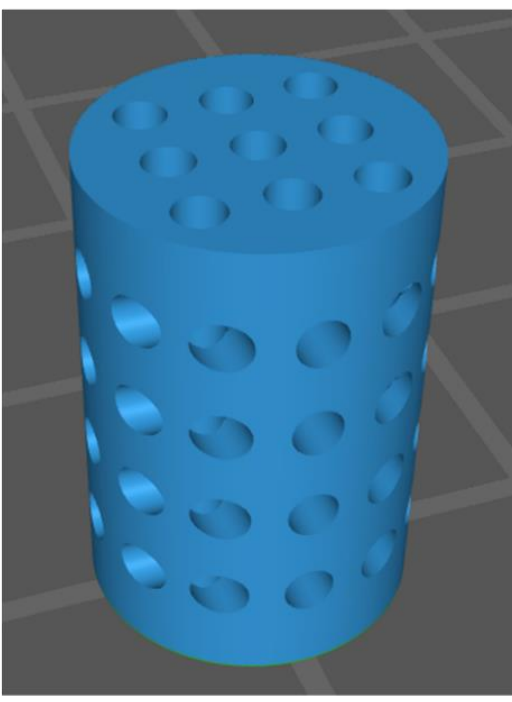

(b)

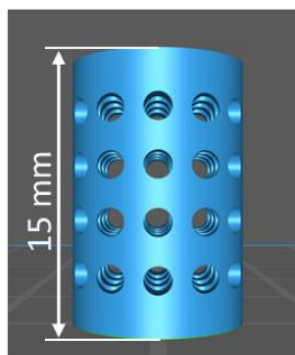

(c)

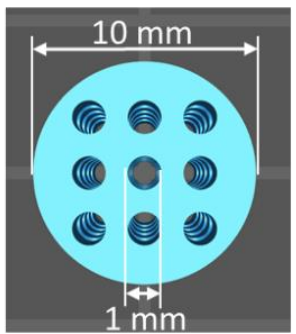

Figure 2. 3D model used to print the HA parts: (a) perspective, (b) front view, (c) top view.

Table 1. MSLA printing parameters profile used for the production of HA scaffolds.

\begin{tabular}{|c|c|c|c|c|c|c|c|c|c|c|}
\hline \multirow[b]{2}{*}{ Parameter } & \multicolumn{3}{|c|}{ Basic information } & \multicolumn{4}{|c|}{ Burn layer (first 6 layers) } & \multicolumn{3}{|c|}{ Normal layer } \\
\hline & $\begin{array}{l}\text { Layer } \\
\text { height } \\
(\mathrm{mm})\end{array}$ & $\begin{array}{c}\text { Down speed } \\
(\mathrm{mm} / \mathrm{min})\end{array}$ & $\begin{array}{l}\text { Delay } \\
(\mathrm{ms})\end{array}$ & $\begin{array}{l}\text { Layers } \\
\text { (number) }\end{array}$ & $\begin{array}{l}\text { Cure } \\
\text { time } \\
(\mathrm{ms})\end{array}$ & $\begin{array}{l}\text { Lift } \\
\text { height } \\
(\mathrm{mm})\end{array}$ & $\begin{array}{l}\text { Up speed } \\
(\mathrm{mm} / \mathrm{min})\end{array}$ & $\begin{array}{l}\text { Cure } \\
\text { time } \\
(\mathrm{ms})\end{array}$ & $\begin{array}{l}\text { Lift } \\
\text { height } \\
(\mathrm{mm})\end{array}$ & $\begin{array}{l}\text { Up speed } \\
(\mathrm{mm} / \mathrm{min})\end{array}$ \\
\hline Value & 0.10 & 150 & 1000 & 6 & 40000 & 8 & 100 & 10000 & 7 & 100 \\
\hline
\end{tabular}

Once the printing platform has been calibrated in the z-axis, the slurry is poured inside of the tank container, and with the help of a plastic spatula, it is homogeneously spread to completely cover the FEP film, which separates the resin from the LCD screen. Then the printing process started constructing the model layer by layer combining the curing of the resin by UV light exposition and the movement of the platform in z-axis following the parameters defined. Figure 3 shows the MSLA process together with the resulting HA bioceramic scaffolds and the tank containing the HA-filled resin. Three different HA scaffold models were designed and printed varying the orientation. Three rotation degrees $0^{\circ}, 45^{\circ}$, and $90^{\circ}$ were applied to the models to study their influence on the quality of the printed parts. All models included supports and rafts to avoid the direct contact of the scaffolds with the printing platform surface. 
One time the printing process finished, each raft supporting the scaffold was detached from the platform surface and the supports were carefully cut with tweezers freeing the HA green body scaffold. Then, the scaffolds were soaked in an ultrasonic bath with isopropyl alcohol (IPA) for 3 minutes. Finally, they were swirled around in the IPA to rinse off the extra uncured resin.

(a)

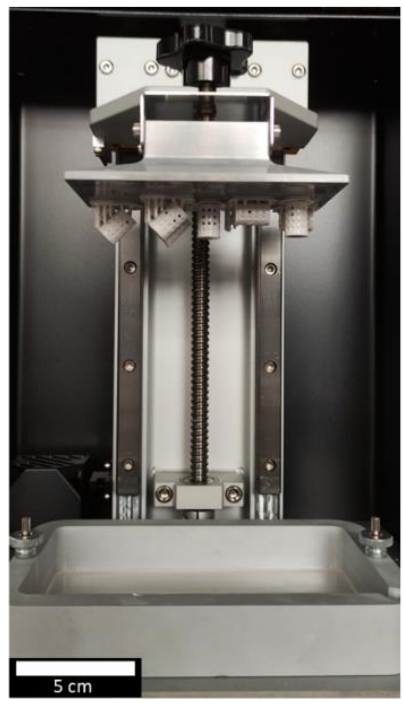

(b)

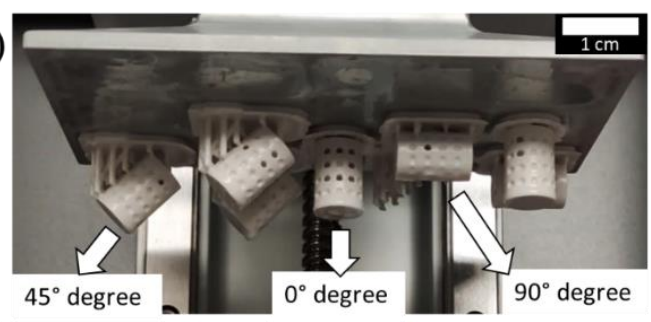

(c)

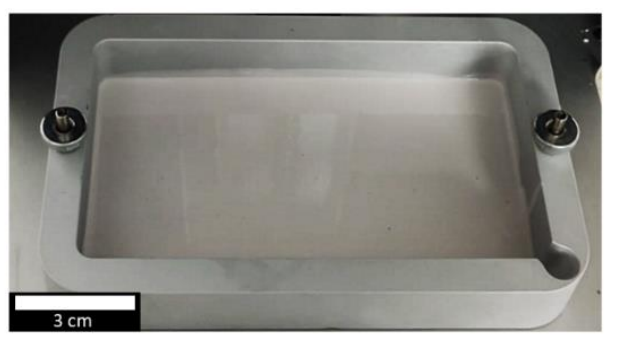

Figure 3. (a) General view of the MSLA process, (b) printing platform with HA scaffolds with three different printing orientations, and (c) HA-filled slurry container.

\subsubsection{Debinding-sintering process}

To obtain HA bioceramic scaffolds free of polymer it was necessary to design a one-step debindingsintering process for the pyrolysis of the cured resin of the printed HA green bodies. Thus, producing in the same process the sintering of the HA particles keeping or improving the mechanical properties of the parts. To prepare the debinding-sintering process, the samples were placed in alumina crucibles and introduced in a muffle furnace Nabertherm ${ }^{\circledR}$ LT $9 / 13$. They were located in a middle height and leaving the same space between the lateral walls inside of the muffle chamber to optimize the heat distribution. The process was carried out in the air and three different temperature phases (or heating ramps) could be distinguished. First, one heating ramp of $1^{\circ} \mathrm{C} / \mathrm{min}$ until $300^{\circ} \mathrm{C}$ followed by another one of $0.2^{\circ} \mathrm{C} / \mathrm{min}$ until $650^{\circ} \mathrm{C}$ performed the 
debinding phase. Then the heating was speeded up at $2^{\circ} \mathrm{C} / \mathrm{min}$ until $1250^{\circ} \mathrm{C}$ and kept constant for two hours to achieve correct sintering of the HA particles. Finally, the furnace was inertially cooled down with an average speed of $-1^{\circ} \mathrm{C} / \mathrm{min}$ to prevent the apparition of cracks due to thermal shocks. The complete temperature pattern followed is described in Figure 4.

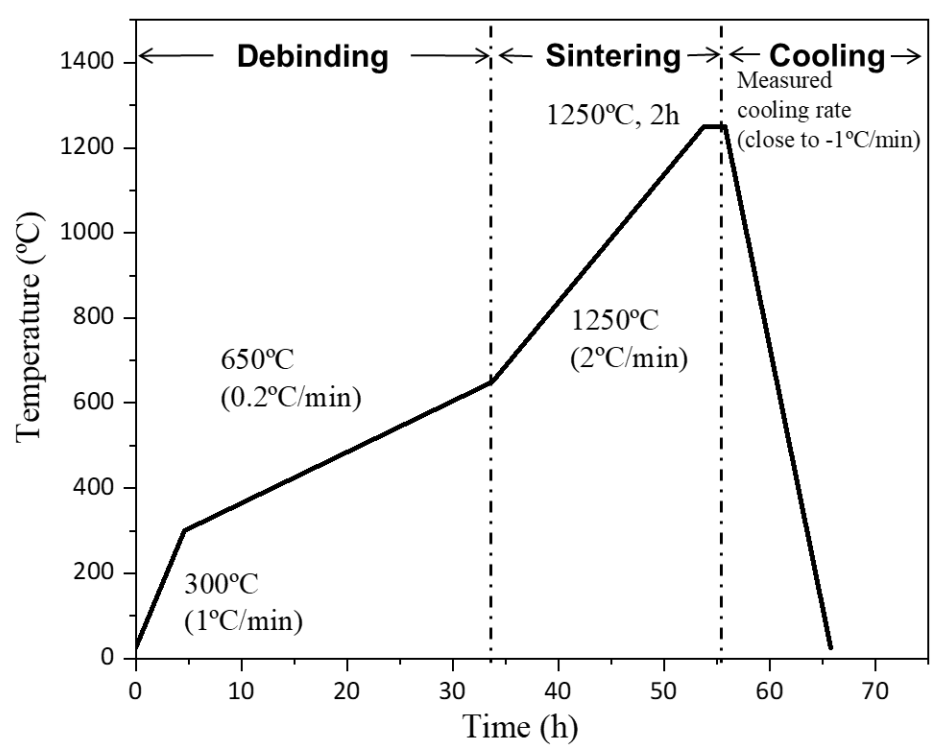

Figure 4. Programmed temperature pattern for the debinding-sintering process of HA scaffolds.

\subsection{Characterization techniques}

\subsubsection{Particle size distribution, porosity and surface area}

A Mastersizer MS 3000 (Malvern Panalytical ${ }^{\circledR}$ ) laser particle size analyser (refractive indexes: 1.63 and 1.33 for particles and water, respectively) was used to analyse the PSD of the HA powders by laser diffraction in suspension. It was not possible to analyse the PSD of the HA powders suspended in the photocurable resin due to a risk of polymerization by the blue light (wavelength: $470 \mathrm{~nm}$ ) used for the measurement. Instead, to simulate their dispersion state they were analysed in aqueous suspension. Ultrapure water used as dispersion medium was produced by the equipment "Purelab Ultra" of VWS (UK) Ltd. Physisorption analysis of powders was quantified in a Micromeritics ${ }^{\circledR}$ TriStar II Plus 3.00 equipment. 
Samples were previously degassed at $40^{\circ} \mathrm{C}$ overnight in a VacPrep ${ }^{\mathrm{TM}} 061$. The data from $\mathrm{BJH}$ pore size distribution desorption was used to determine exactly the average pore volume. Nitrogen adsorptiondesorption isotherm properties were used to calculate the specific surface area through the BET method.

\subsubsection{Turbidimetry analysis}

Turbiscan stability index (TSI) (a Turbiscan specific parameter for stability comparison) and backscattered (BS) profiles of the different HA-filled slurries were obtained using a Turbiscan LAB ${ }^{\mathrm{TM}}$ $\left(\right.$ Formulaction $\left.^{\circledR}\right)$. The light source scanned the sample tube at 2 min intervals from top to bottom and measured the percentage of light BS or transmitted during a $3 \mathrm{~h}$ period at $25^{\circ} \mathrm{C}$ to cover the total time needed for the printing process of the green bodies (about two hours). Longer analyses (up to 66 hours) were performed to evaluate the time of destabilization. The analysis of the stability was performed as a variation of BS profiles as a function of time at the totality of the sample height. The curves obtained allow a better quantification of their dispersion stability and identify the phenomena taking place in the samples (sedimentation, flocculation...). The overall stability of slurries was examined using the TSI parameters calculated by the Turbiscan software using the formulae below [32]:

$$
\begin{gathered}
B S=\frac{1}{\sqrt{\lambda *}} \\
\lambda *\left(\varphi, x_{50}\right)=\frac{2 x_{50}}{3 \varphi(1-g) Q s} \\
T S I=\sqrt{\frac{\sum_{i=1}^{n}(\chi i-\chi B S)^{2}}{n-1}}
\end{gathered}
$$


where $\lambda^{*}$ is the photon transport mean free path in the sample, $\varphi$ is the volume fraction of particles, $x_{50}$ is the mean diameter of particles, $g$ and $Q s$ are optical parameters given by the Mie theory. $\chi i$ is the average backscattering for each minute of measurement, $\chi B S$ is the average $\chi i$, and $\mathrm{n}$ is the number of scans.

\subsubsection{Rheological analysis of suspensions}

The rheological behaviour of the organic resin and HA-filled suspensions at different concentrations was examined using a TA Instruments AR2000 rheometer. A 40mm crosshatched parallel plates system was used as geometry $(\mathrm{gap}=1300 \mu \mathrm{m})$. Flow curves of each sample were produced at a constant plate temperature of $25^{\circ} \mathrm{C}$ controlled by a Peltier plate. An increasing shear rate (steady-step flow step) in the range of 1-100 $\mathrm{s}^{-1}$ was used taking ten points by decade.

\subsubsection{Chemical and structural analyses}

The calcium phosphate crystalline phases in the sintered scaffolds were detected by X-ray diffraction (XRD) analysis at different temperatures by using a BRUKER's X-ray diffractometer D8 Advance system with $\mathrm{Cu} \mathrm{K} \alpha$ radiation (wavelength $\lambda=0.15406 \mathrm{~nm}$ ). Equipped with a high-temperature sample stage, a LYNXEYE XE-T detector (energy resolution of $0.38 \mathrm{keV}$ ) and a nickel filter at $40 \mathrm{kV}$ and $40 \mathrm{~mA}$. Samples were analysed in a $2 \theta$ range between $20^{\circ}$ and $60^{\circ}$, a step of $0.03^{\circ} 2 \theta$ and a time per step of $0.2 \mathrm{~s}$. Measurements were performed at key temperature values of the temperature pattern used for the debindingsintering process.

Sintered HA scaffold pieces obtained after the uniaxial compression test were hand-crushed using a mortar and pestle to produce a homogeneous powder. Powdered samples were examined by Fourier transform infrared spectroscopy (FTIR) analysis in a spectrometer FTIR iS50 using the KBr pellet preparation method to determine its chemical composition. Approximately $9 \mathrm{mg}$ of sample powder are transferred to a clean mortar, then a weighed amount of dry $\mathrm{KBr}$ powder $(300 \pm 5 \mathrm{mg})$ is added and mixed gently to produce a homogenous mixture, followed by compression at $6000 \mathrm{~kg}$ for the sample disk 
preparation. FTIR spectra were recorded in the $4000-400 \mathrm{~cm}^{-1}$ wavenumber range with a step width of 0,48 $\mathrm{cm}^{-1}$ for each sample disk.

\subsubsection{Analysis of macro-structural properties of HA scaffolds}

The morphology of HA powders, scaffolds surface, and the cross-section were examined with a scanning electron microscope (SEM) LEO 435 VP (Leica®) equipped with a Ge detector (Imix-PC, Princeton gamma-tech) and a metallization of the ceramic surface was applied with a thin film of silver employing a Scancoat Six sputter coater. HA scaffolds surface was polished in the X, Y plane to analyse the crosssection of the parts. Firstly, the samples were coarse, medium, and fine ground using a Minitech 263 and silicon carbide papers of different grit sizes to reach the region of interest (about $3 \mathrm{~mm}$ depth in the $\mathrm{X}, \mathrm{Y}$ plane). Then, a DiaPro alcohol-based $3 \mu \mathrm{m}$ high concentration diamond suspension from Struers ${ }^{\circledR}$ and a cloth paper were used in a Struers ${ }^{\circledR}$ Tegrapol 25 to obtain a better surface finishing. ImageJ was used for the processing of the images [33].

High-resolution X-ray micro-computed tomography (CT) analyses of the printed parts were performed in a Nanotom ${ }^{\circledR} 180$ Phoenix - GE equipped with a $180 \mathrm{kV} / 15 \mathrm{~W}$ high-power nanofocus X-ray tube $(9 \mu \mathrm{m}$ voxel size, resolution). This analysis allowed us to have a 3D perspective of the scaffold and evaluate their total porosity (microstructural porosity of sintered HA and lattice porosity of the scaffold architecture). The data sets were reconstructed and three-dimensionally visualized using VGStudio MAX 4.4 software.

The geometrical density of the scaffolds was calculated from the mass and dimensions of at least ten samples. The theoretical density of HA $\left(3.16 \mathrm{~g} . \mathrm{cm}^{-3}\right)$ was used as a reference to calculate the total volume fraction of porosity. The open porosity of the scaffolds was measured by Archimedes' method using distilled water as liquid.

The shrinkage of the scaffolds after the debinding-sintering process was studied by comparison of the scaffold's dimensions before and after the thermal treatment. Three measurements of the height at different 
rotation angles (every $60^{\circ}$ ) were used to calculate the height average and the z-axis shrinkage of each printed part. For the diameter average and the $\mathrm{x}$, $\mathrm{y}$ axes shrinkage, nine measurements were carried out (three measurements at three different heights of the part: bottom, middle, top) also at different rotation angles (every $\left.60^{\circ}\right)$.

Mercury intrusion porosimetry was used to analyse the total connected porosity, volume of pores, and pore size distribution $>50 \mathrm{~nm}$ (meso- and macropores) of the printed scaffolds. These analyses were performed in an AutoPore IV 9500.

\subsubsection{Mechanical properties}

The compressive strength of the sintered HA scaffolds with different orientations was measured in a universal mechanical testing press HOUNSFIELD H25KS (Tinius Olsen ${ }^{\circledR}$, USA). A stainless-steel compression miniature load cell T22-252 U4000 with a range of $25 \mathrm{kN}$ was used to measure the shear force at a crosshead speed of $0.10 \mathrm{~mm} / \mathrm{min}$ at room temperature. At least ten samples $(\mathrm{N}=10)$ from the same batch were evaluated to calculate the mean values and the standard deviation. The procedure followed is described in the standard ISO-13175:2012. To measure the maximum compressive load taken by the specimens, the load given by the testing press in the direction shown in Figure 5, was increased till the complete failure of the specimens due to crack propagation.

(a)

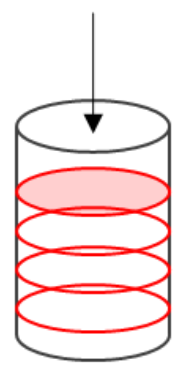

(b)

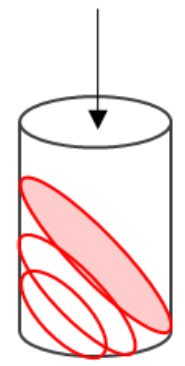

(c)

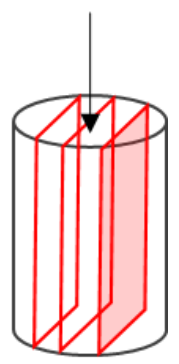

Figure 5. Direction of loading (black arrows) to printing orientation in compressive testing. (a) $0^{o}$, (b)

$$
45^{\circ} \text {, and (c) } 90^{\circ} \text {. }
$$

\subsubsection{Dissolution rate and $\mathrm{pH}$ change}


In vitro dissolution rate of the sintered scaffolds, $\mathrm{Ca}^{2+}$ ion release, and $\mathrm{pH}$ change of the dissolution medium were evaluated following the procedure also described in the standard ISO-13175:2012. Three samples of HA scaffold (printed at orientation $0^{\circ}$ ) were introduced in three flasks of $0.05 \mathrm{M}$ TRIS buffer solution after nitric acid addition for $\mathrm{pH}$ adjustment at $7.3 \pm 0.1$ at $37 \pm 1^{\circ} \mathrm{C}$. The three solution flasks were placed on a plate agitator with a rotation speed of 200rpm for $24 \mathrm{~h}, 48 \mathrm{~h}$, and $72 \mathrm{~h}$ respectively. The ratio of initial material mass to total dissolution media volume was kept constant at $4.0 \mathrm{mg} \cdot \mathrm{ml}^{-1}$. $\mathrm{pH}$ was measured after $0 \mathrm{~h}, 24 \mathrm{~h}, 48 \mathrm{~h}$, and $72 \mathrm{~h}$ of immersion. Thus, ensuring that the initial $\mathrm{pH}$ value did not vary more than 0.3 during the testing. The $\mathrm{Ca}^{2+}$ ion content of the solutions was analysed by ICP/AES in an inductively coupled plasma atomic emission spectrometry (ICP/AES, Ultima2R HORIBA ${ }^{\circledR}$ ). The concentrations versus time curves were determined. The test was repeated three times $(\mathrm{N}=3)$ to calculate the mean values and the standard deviation.

\subsubsection{Thermal analysis}

Thermogravimetric (TGA) and differential scanning calorimetry (DSC) analyses were performed to study and confirm the mass loss evolution of the products and the energy transferred to or from the sample during the debinding-sintering process. It was performed by introducing a small portion $(0.02 \mathrm{~g})$ of the ceramic green body in a Setsys Evolution TG92 (Setaram $\left.{ }^{\circledR}\right)$ and applying a heating ramp of $10^{\circ} \mathrm{C} / \mathrm{min}$ until $1250^{\circ} \mathrm{C}$ in air. 


\section{Results and discussion}

\subsection{HA-filled suspension preparation}

This section involves the analysis of the three different HA powders ( $\left.\mathrm{HA}_{\text {Initial }}, \mathrm{HA}_{\mathrm{PBM}}, \mathrm{HA}_{\mathrm{SBM}}\right)$ properties and the evaluation of their influence on the final HA-filled suspension stability and rheological behaviour. Only the HA-filled suspension showing the best attributes for its application in an MSLA printer will be finally used for the preparation of the HA scaffolds.

\subsubsection{HA powders properties comparison}

Particle size distribution (PSD) and scanning electron microscopy (SEM) micrographs of each of the three $\mathrm{HA}$ powders $\left(\mathrm{HA}_{\text {Initial }}, \mathrm{HA}_{\mathrm{PBM}}, \mathrm{HA}_{\mathrm{SBM}}\right)$ used for the preparation of the photocurable organic suspensions in the present study are shown in Figure 6. Clear differences in the particle median sizes $\left(\mathrm{x}_{50}\right)$ and spans can be observed between the three powders. $\mathrm{HA}_{\text {Initial }}$ is composed of mainly one population composed of agglomerates produced by the calcination of the powder with an $\mathrm{x}_{50}$ of $25 \mu \mathrm{m}$.

The use of specific milling conditions during the SBM process (wet grinding) of this powder causes the particle size reduction giving as result the $\mathrm{HA}_{\mathrm{SBM}}$ powder. The product particle size reached a $\mathrm{x}_{50}$ of $2.2 \mu \mathrm{m}$ measured in aqueous suspension (without any dispersant). The powder is formed by two particles populations, one with submicronic size $(0.7 \mu \mathrm{m})$ and another one close to $2.5 \mu \mathrm{m}$.

The application of a lower energetic milling process as PBM produces a less intense particle size reduction of the $\mathrm{HA}_{\text {Initial }}$ powder ( $\mathrm{x}_{50}$ of $12 \mu \mathrm{m}$ (measured in aqueous suspension)) [34]. As result, the PSD of the HАРвм powder becomes more disperse with two particles populations, one of $20 \mu \mathrm{m}$ and another one of $2.5 \mu \mathrm{m}$. Regarding the particles form, all three powders show irregular shapes with no noticeable variations between them. 


\section{(a)}

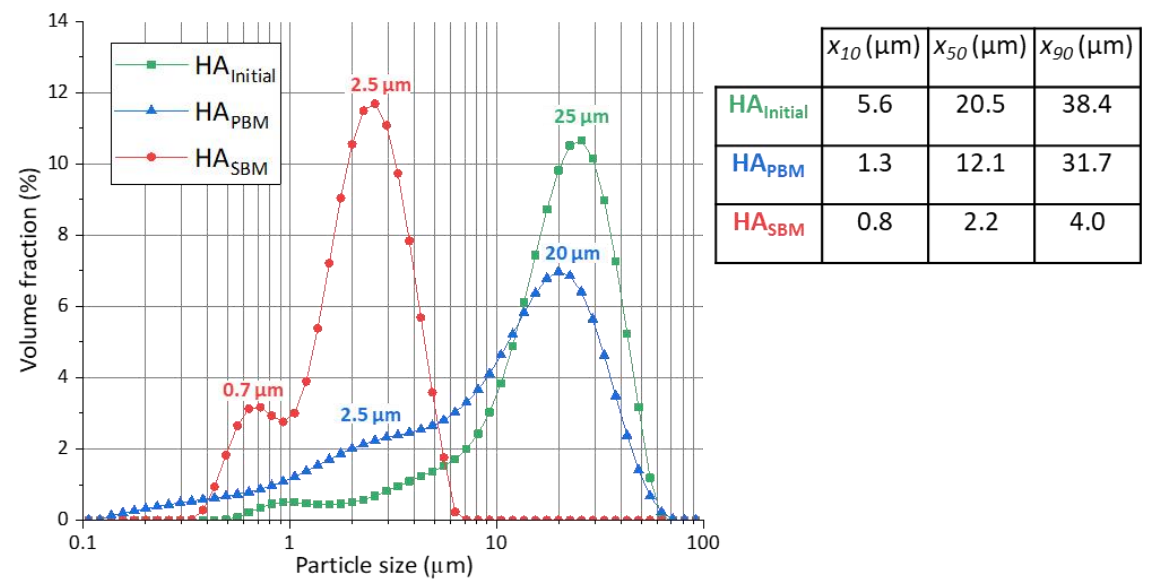

(b)

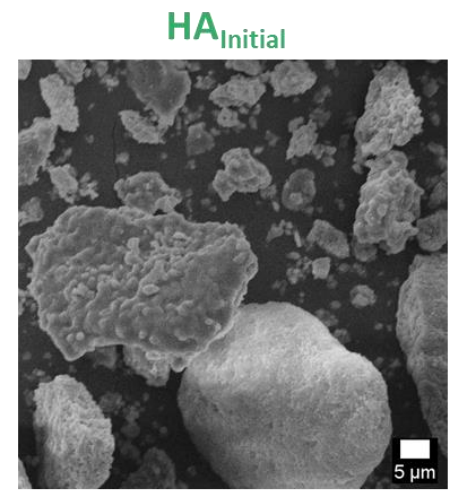

(c)

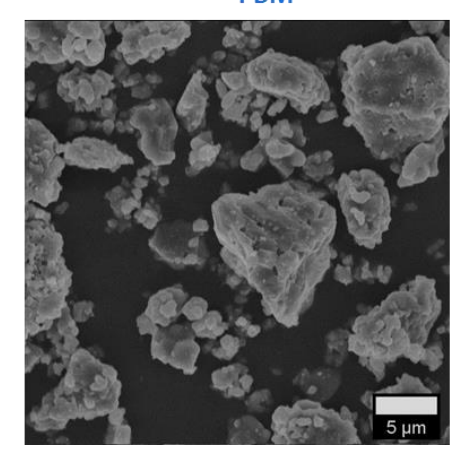

(d)

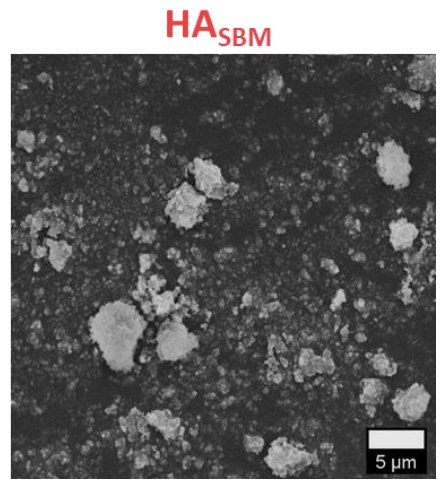

Figure 6. (a) PSD and SEM micrographs of HA powders used in the study: (b) $H A_{\text {Initial, }}$ (c) $H A_{P B M}$, (d) $H A_{S B M}$.

It was observed that the surface area of the initial HA powder $\left(6.9 \mathrm{~m}^{2} \cdot \mathrm{g}^{-1}\right)$ is increased after undergoing SBM process $\left(23.8 \mathrm{~m}^{2} \cdot \mathrm{g}^{-1}\right)$ while after PBM process the surface area diminished $\left(4.6 \mathrm{~m}^{2} . \mathrm{g}^{-1}\right)$. Logically, an increase in the surface area of the powder would be expected after both milling processes because of a reduction of particle size [35]. However, the obtained values show the contrary in the case of the PBM process. This phenomenon was already observed by Granados-Correa et al. [34] in their study on the ball milling effect on tribasic calcium phosphate powders. The explanation offered by Granados-Correa et al. referred to the fact that apart from the calcium phosphate grains breakage and size diminution, the ball mill strikes induced compression of the resulting particles reducing their pore volume. It results then in a decrease in the total surface area of the powder. This theory gains reliability after the analyses of the total pore volume of powders. The values obtained were $0.017,0.015$, and $0.112 \mathrm{~cm}^{3} \cdot \mathrm{g}^{-1}$ for $\mathrm{HA}_{\text {Initial }}, \mathrm{HA} \mathrm{ABM}_{\mathrm{PBM}}$, and 
$\mathrm{HA}_{\mathrm{SBM}}$ respectively, confirming the unforeseen decrease of the specific surface area of the PBM treated powder.

\subsubsection{Suspension's stability and rheology behaviour comparison}

Before start preparing the HA-filled suspensions the effect of the addition of a $25 \%$ vol. of PEG200 (maximum concentration suitable without compromising the reactivity of the resin) on the rheological behaviour of the commercial organic resin base was evaluated. Figure 7 a shows the comparison between the flow curves obtained for the Dentifix ${ }^{\circledR}$ resin base before and after the addition of a $25 \%$ vol. of PEG200 compound acting as a reactive diluent. Both samples showed a shear-thinning character with a decrease in ramp average viscosity from $\sim 0.076$ Pa.s to $\sim 0.065$ Pa.s after the addition of the active diluent PEG200. This proportion resin/diluent was used for the preparation of all HA-filled slurries.

The flow curves obtained for suspensions containing a $20 \%$ vol. of different HA powders $\left(\mathrm{HA}_{\text {Initial, }}\right.$ $\mathrm{HA}_{\mathrm{PBM}}, \mathrm{HA}_{\mathrm{SBM}}$ ) with different median particle sizes is shown in Figure $7 \mathrm{~b}$. The comparison of the three viscosity-shear rate curves shows that the suspension with the highest viscosity was obtained when using the $\mathrm{HA}_{\mathrm{SBM}}$ powder. It was already explained that for similar sample volume and solid concentration, smaller particle size means a higher number of particles present in the suspension, which can show Brownian motion acting against an applied shear [36,37]. Since this suspension containing HAsBM showed viscosity values at a low shear rate over the maximum viscosity limit for its utilization in stereolithography (5 Pa.s), it was discarded as feedstock for the printing process. When comparing the viscosity-shear rate curves obtained by $\mathrm{HA}_{\text {Initial }}$ and $\mathrm{HA}_{\mathrm{PBM}}$ suspensions no big differences were observed, which could be due to the less significant variation between their PSDs. 
(a)

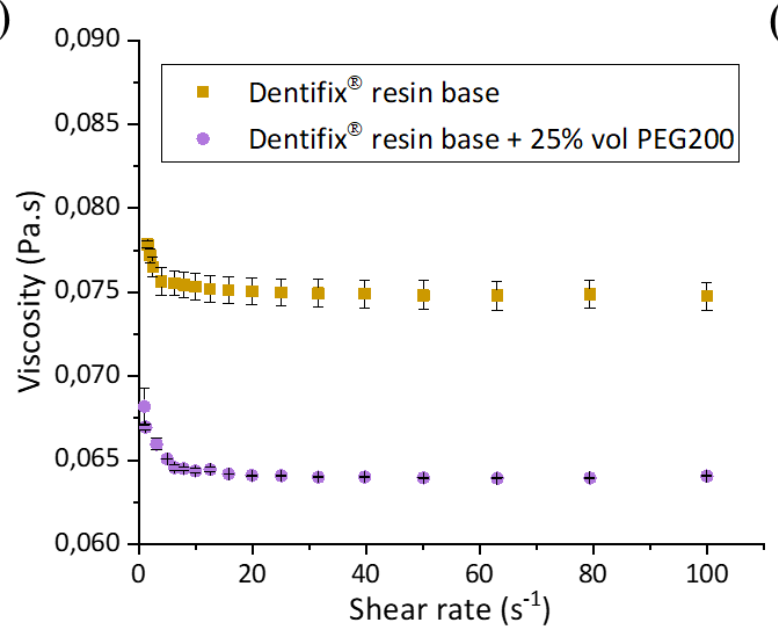

(b)

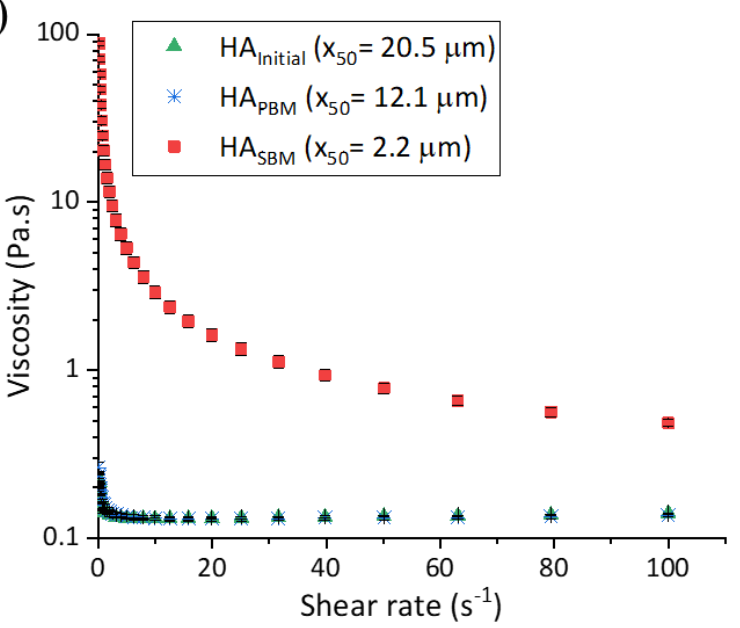

Figure 7. (a) The flow data for Dentifix ${ }^{\circledR}$ resin base with and without the addition of $25 \%$ vol. of PEG200 as diluent. (b) The flow data for photocurable suspensions filled a $20 \%$ vol. with different HA powders $\left(H A_{\text {Initial }}, H A_{P B M}, H A_{S B M}\right)$ with different median particle sizes $\left(H A_{\text {Initial }}\right.$ and $H A_{P B M}$ show very close viscosities).

A comparison between the stabilities of the three types of HA-filled suspensions was carried out.

Figure 8 shows the BS profiles of the HA-filled suspensions for three hours. The suspensions prepared with $\mathrm{HA}_{\mathrm{SBM}}$ showed the highest stability since it showed almost no alteration of the BS profile during the whole time of analysis. The slurries containing $\mathrm{HA}_{\text {Initial }}$ and $\mathrm{HA}_{\text {PвM }}$ showed both particles sedimentation (BS increases at the bottom of the tube) and clarification (BS decreases at the top) phenomena, however, it was less pronounced in the second case. The differences between the stabilities can be due to different factors that can affect the rate of sedimentation of the prepared suspensions, such as the particle size and the viscosity of the suspensions. It is known that the smaller the particle size and higher the viscosity of a suspension, the slower will be the sedimentation rate of the particles [38]. Which, could explain the results obtained. 
(a)

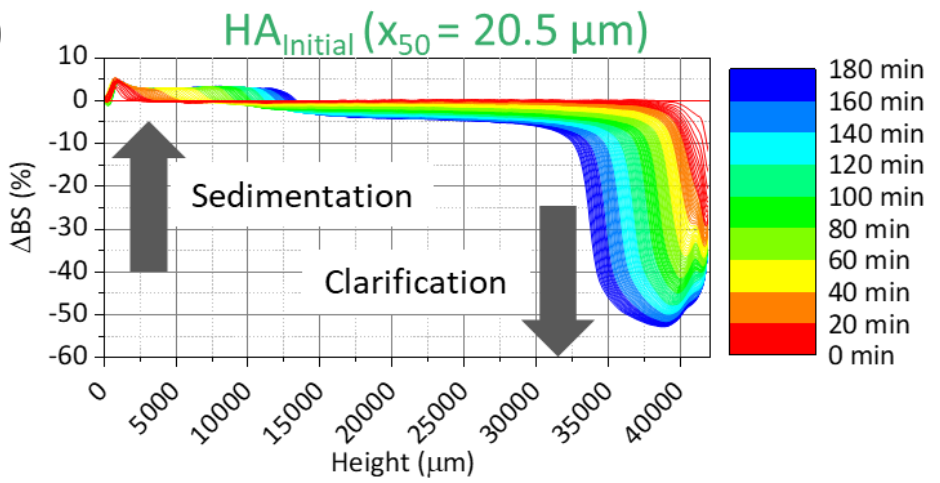

(b)

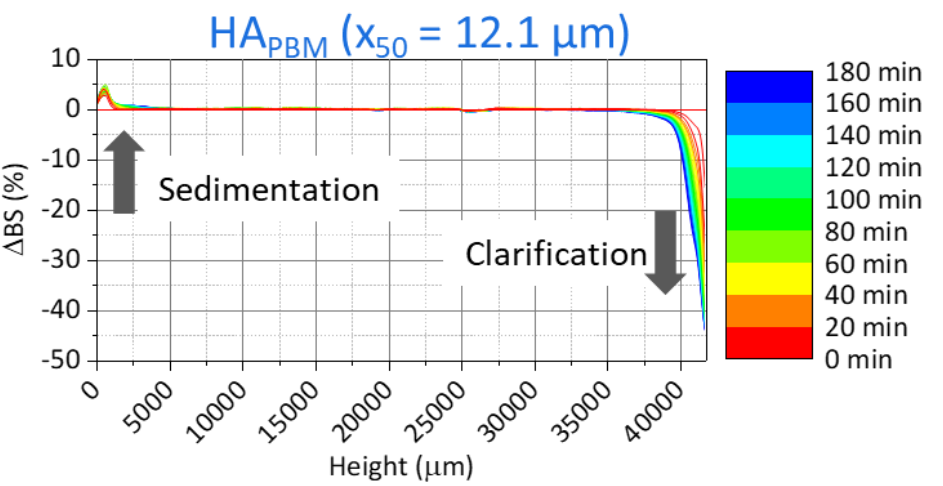

(c)

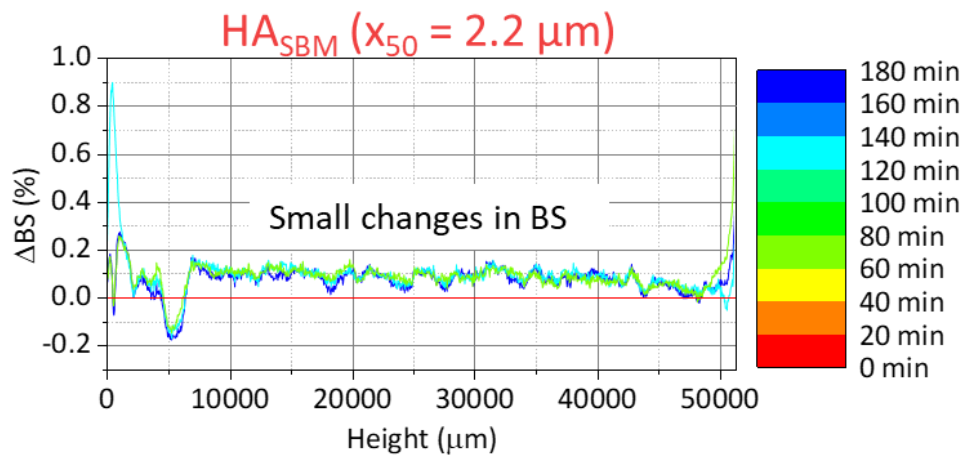

Figure 8. Backscattering profiles of HA-filled suspensions prepared with different HA powders. (a)

$$
H A_{\text {Initial }} \text { (b) } H A_{P B M},(c) H A_{S B M}\left(T=25^{\circ} \mathrm{C}\right) \text {. }
$$

As mentioned in the introduction, the overall stability of slurries described as a TSI value lower than 2 was pursued for the duration of the HA parts fabrication. Figure 9 shows the evolution of the TSI values obtained for the HA-filled slurries as a function of time.

The suspension containing the $\mathrm{HA}_{\text {Initial }}$ powder showed the worst stability (TSI $\cong 2.75$ ) after three hours of the three suspensions. The highest stability was obtained by the slurry with HA 
by the smallest particles, whose TSI value remained almost unaltered for the whole duration of the analysis. $\mathrm{HA}_{\mathrm{PBM}}$ suspension showed intermedium stability with a TSI value close to 1.48 after three hours.

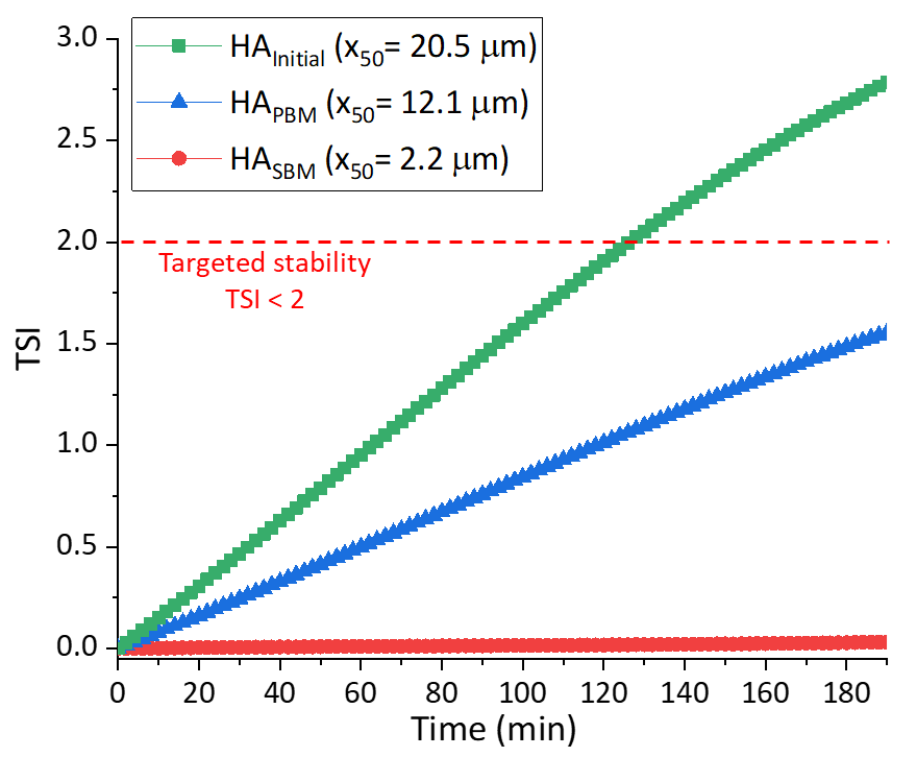

Figure 9. TSI values of HA-filled suspensions as a function of time $\left(T=25^{\circ} \mathrm{C}\right)$.

The previous stability and rheological behaviour analyses allowed us to compare the properties of the suspensions prepared with different HA powders. The order for the stabilities observed for the three kinds of slurries was $\mathrm{HA}_{\mathrm{SBM}}>>\mathrm{HA}_{\mathrm{PBM}}>\mathrm{HA}_{\text {Initial }}$ with a clear difference between the powder of finer particle size (HA $\left.\mathrm{ABM}_{\mathrm{SB}}\right)$ to the others two. However, the study of the rheology of the suspensions showed the following viscosity order for the slurries $\mathrm{HA}_{\mathrm{SBM}} \gg \mathrm{HA}_{\mathrm{PBM}} \cong \mathrm{HA}_{\text {Initial. }}$. The high viscosity showed by the suspension prepared with $\mathrm{HA}_{\mathrm{SBM}}$ (low shear rate viscosity > $5 \mathrm{~Pa} . \mathrm{s}$ ) hindered the homogeneous spread of the suspension to be employed as feedstock in the MSLA printer. Indeed, the apparatus employs the vertical movement of the printing stage to refill with suspension the space between the stage and the LCD screen. The poor flow of this suspension makes impossible this indispensable refilling step. For this reason, the use of this powder as filler was discarded for the production of photocurable HA-filled suspension feedstock. The suspension showing the most favourable results on suspension rheology (low shear viscosity $<5$ Pa.s) and stability $(\mathrm{TSI}<1.5)$ was the prepared with the powder HAPBM. For this reason, only this powder was considered for 
the preparation of the highly-loaded photocurable HA-filled resins that will be used as feedstock for the production of HA scaffolds via the MSLA process.

The powder concentration is known to be an important factor influencing the degree of shrinkage during the posterior debinding process. The higher the solid loading the lower the shrinkage, but a too high solid loading makes difficult the debinding process due to the impeding of the gases diffusion [24]. It is then of special interest to know the maximum concentration reachable without affecting negatively the handling and processability of the suspension as well as the debinding process. To be sure that the properties for the suspension are still valid for its utilisation at higher concentration, a study of the influence of the solid volume concentration of $\mathrm{HA}_{\mathrm{PBM}}$ powder in the slurry properties was carried out.

Figure 10a shows the comparison between viscosity-shear rate curves for different HA-filled suspensions containing $\mathrm{HA}_{\mathrm{PBM}}$ concentrations ranging 20-50 vol. \%. A shorter flow ramp was produced for the sample at 50 vol. \% due to geometry limitations measuring high concentrated suspensions at high shear rates. However, this ramp was enough for the comparison of the rheological behaviour between suspensions. Even if an increase in the suspension viscosity is visible when a higher solid volume concentration (50 vol. \%) is used for the preparation of the slurries ( 2 Pa.s at a shear rate of $\left.1 \mathrm{~s}^{-1}\right)$, it did not exceed the settled maximum limit of 5 Pa.s.

Regarding the suspension behaviour at different solid volume concentrations, at a concentration of $40 \%$, we observed a transition from shear-thinning to a shear-thickening character. The behaviour of suspension changes at different volume fractions [39]. At higher concentrations, an increase of the shear rate may lead to the formation of particles clusters and jamming showing a shear thickening behaviour. The transition from shear-thinning to shear-thickening is sample-specific and is known to be controlled by factors such as particle shape and particle size [40].

Collisions between particles present in a suspension are expected. Thus, they act as obstacles, and their friction demands further shear force. The higher is the solid volume fraction, the more significant the ongoing particle-particle interactions increasing the force needed to shear the suspension. This is defined 
as the 'crowding' effect which has been described by many semi-empirical models, however, the most popular equation was the one developed by Krieger and Dougherty (K-D) [41]:

$$
\eta_{r}=\frac{\eta}{\eta_{\text {medium }}}=\left(1-\frac{\varphi}{\varphi_{m}}\right)^{-[\eta] \varphi_{m}}
$$

Where $\eta_{r}$ is the relative viscosity between $\eta$ the viscosity of the suspension and $\eta_{\text {medium }}$ the viscosity of the medium. $\varphi$ is the volume fraction of solids in the suspension, $\varphi_{m}$ is the maximum volume fraction of solids in the suspension ( 0.64 for spheres of similar size), and $[\eta]$ is the intrinsic viscosity (2.5 for spheres). Different authors have used a modified version of the K-D equation which includes the effective packing factor of ceramic powder, $\beta$, which can be determined experimentally achieving a better fitting for ceramic suspensions [42,43]. This modified version is written as follows:

$$
\eta_{r}=\left(1-\frac{\beta \varphi}{\varphi_{m}}\right)^{-[\eta] \varphi_{m}}
$$

Since this analysis for correlation was out of the scope of the paper no further effort was employed on the determination of the effective packing factor of the specific suspensions under study. Instead, to perform a preliminary evaluation of the fits a value of $\beta=1.58$ was used. This value was previously determined by Chu and Halloran [43] for HA-filled non-aqueous ceramic suspensions. A maximum volume fraction of solids in the suspension, $\varphi_{m}=0.51$ was considered for the calculation. The fitting for both equations (K-D and modified K-D) to the obtained experimental values is shown in Figure 10b. It can be observed that, still, a deeper study could be performed to obtain a better fitting between calculated and experimental values. However, between both K-D equations, the modified version showed a smaller residual sum of squares (313.78) indicating a better fit to our data. 
(a)

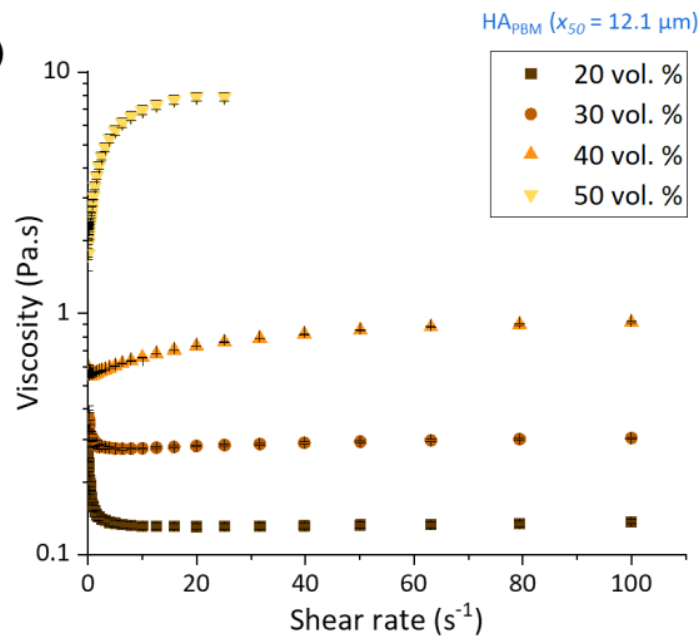

(b)

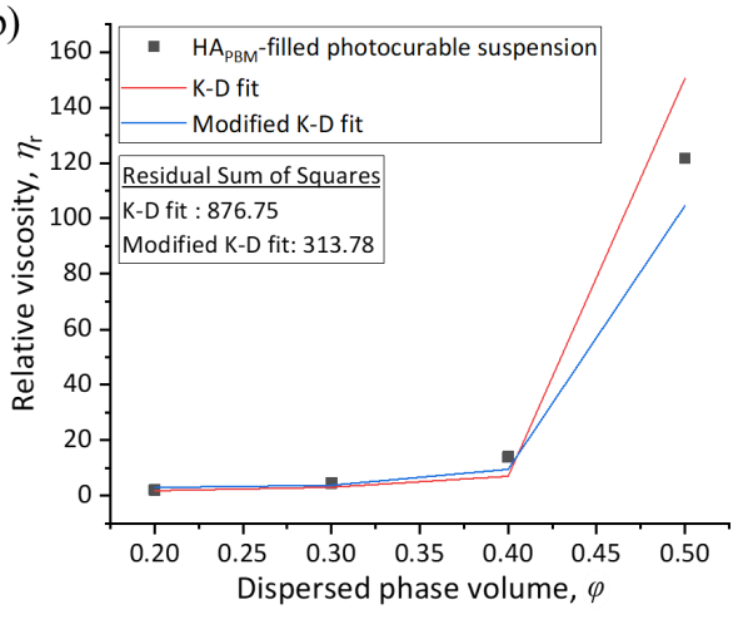

Figure 10. (a) The viscosity-shear rate curves obtained for several HA-filled suspensions containing different $H A_{P B M}$ concentrations. (b) Krieger-Dougherty (red line) and modified Krieger-Dougherty (blue line) fits for relative viscosities in $H A_{P B M}$-filled suspensions.

The study of the stability of the suspension containing $\mathrm{HA}_{\mathrm{PBM}}$ powder at $50 \mathrm{vol} . \%$ is shown in Figure 11. The phenomena occurring during the duration of the test in the suspension at $50 \mathrm{vol} . \%$ was examined by analysing its BS profile shown in Figure 11a. A sedimentation behaviour was observed for this suspension although less pronounced than the one observed for the suspension at a concentration of 20 vol. $\%$ already seen in Figure 8c. Figure 11a compares the TSI values of the HAPBM suspensions at 20 and 50 vol. \% concentrations. A lower TSI value (0.85) was observed for the slurry with a higher concentration after 180 minutes. It indicates higher stability compared with the slurry at a lower concentration, which shows a higher TSI (1.48) for the same duration. It took about $5 \mathrm{~h}$ for the suspension at 20 vol. $\%$ to exceed the fixed limit of destabilization (TSI $=2$ ), while the suspension at 50 vol. $\%$ needed almost $10 \mathrm{~h}$ to reach this value. This confirms the principle for high concentrated suspensions showing a slow sedimentation rate [38]. The influence of sediment particle concentration on the settling velocity has been amply investigated [44,45]. It has been demonstrated that the settling velocity is lower at a higher concentration by a factor usually given by the widely used semiempirical equation offered by Richardson and Zaki [46]: 


$$
\frac{w_{m}}{w}=(1-\varphi)^{n}
$$

Where $w\left(m . s^{-1}\right)$ is the terminal settling velocity of an individual particle in a fluid; $\varphi$ is the sediment volumetric concentration (-); and $w_{m}\left(m \cdot s^{-1}\right)$ is the settling velocity of sediment particles dispersed at the volumetric concentration $\varphi . n$ is an empirically determined exponent dependent on the particle Reynolds number $R$. Researchers have made an effort determining n-values to increase the accuracy of the prediction associated with equation (5) [45].

The terminal velocity of an individual particle of HA was calculated by stokes' law ( $\mathrm{w}=1.04 \cdot 10^{-2} \mathrm{~m} . \mathrm{s}^{-}$ $\left.{ }^{1}\right)$. Then, following the equation (6), the terminal settling velocities for the HАРвM particles dispersed in the photocurable resin (including diluent) at different concentrations $\left(w_{m}\right)$ were obtained. The ratios between both velocities $\left(w / w_{m}\right)$ at different volume concentrations are shown in Table 2. For the calculation, a laminar regime $(R<0.2)$ was considered with an n-value of 4.65 . It can be observed how, for example, the settling velocity of the particles is delayed by $2,51 \cdot 10^{1}$ with a concentration of $50 \mathrm{vol}$. $\%$. Then, the decrease in settling velocity with the increase in concentration was demonstrated. 
Table 2. Settling velocities of particles dispersed at different volume concentrations. $n=4.65, w=$ $1.04 \cdot 10^{-2} \mathrm{~m}^{-1} \mathrm{~s}^{-1}$ (obtained by stokes' law)

\begin{tabular}{|c|c|}
\hline Volume concentration (\%) & $w / w_{m}$ \\
\hline 20 & 2.82 \\
\hline 30 & 5,25 \\
\hline 40 & $1,08 \cdot 10^{1}$ \\
\hline 50 & $2,51 \cdot 10^{1}$ \\
\hline
\end{tabular}

(a)

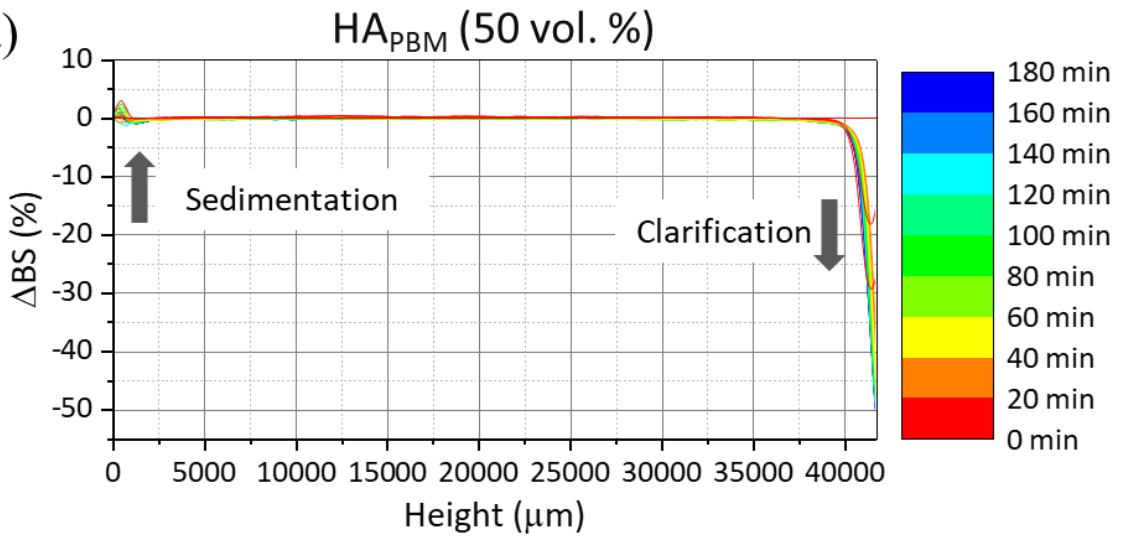

(b)

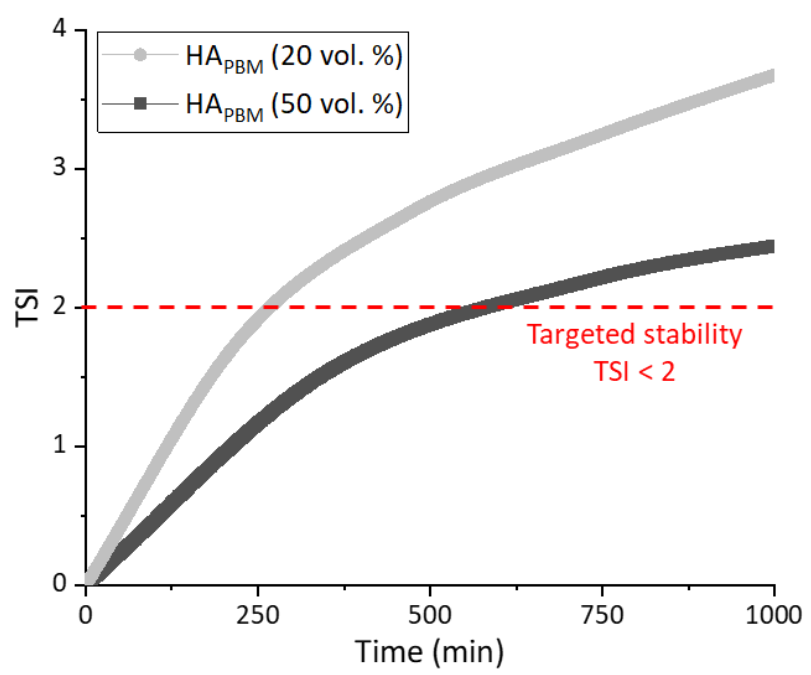

Figure 11. (a) Backscattering profile of $H A_{P B M}$ suspension at $50 \%$ vol. $\left(T=25^{\circ} \mathrm{C}\right)$. (b) TSI values of $H A_{P B M}$ filled suspensions at different concentrations as a function of time $\left(T=25^{\circ} \mathrm{C}\right)$. 
We concluded this study on the tailoring of HA powders and their influence on the suspension's properties (target properties: viscosity $<5$ Pa.s at a shear rate $1 \mathrm{~s}^{-1}$ and TSI $<2$ for the duration of the process) by choosing the $\mathrm{HA}_{\mathrm{PBM}}-$ loaded suspension at 50 vol. \% for its use in the MSLA process.

\subsection{Printing of HA scaffolds}

The suspension containing a 50\% vol. of $\mathrm{HA}_{\mathrm{PBM}}$ was employed for the production of the HA scaffolds following the MSLA printing and debinding-sintering procedures already described. This section will be then focused on the characterization of the parts produced. The composition of the parts will be analysed to discard any alteration of the HA phases during the process. The morphology of the parts will be evaluated through the comparison with the 3D model to analyse the possible shrinkage due to the debinding-sintering process. The bioactivity will be examined through the standard analysis of the dissolution rate of the part. Then the mechanical properties of the parts will be assessed to evaluate the anisotropy behaviour caused due to change in build orientation.

\subsubsection{Composition}

To confirm the crystalline phases of the sintered scaffolds XRD analyses were carried out. The analyses were performed at different temperatures reproducing the debinding-sintering temperature pattern to study the evolution of the phase. Figure 12 shows the in-situ XRD diffractogram of the HA scaffold obtained at different temperatures under air atmosphere. The absence of secondary crystalized calcium phosphate phases was confirmed and only the stoichiometric hydroxyapatite phase (JCPDS 00-009-0432) was observed before, during, and after the debinding-sintering process [47]. This result confirms the thermal stability reported for HA in literature $[48,49]$, indicating stability up to $1350^{\circ} \mathrm{C}$ when it starts to decompose into other calcium phosphate phases such as tricalcium phosphate, and tetracalcium phosphate that could affect negatively the properties of the printed parts (different dissolution rates in physiological conditions, 
uneven grain growth...) [50]. It is known that the densification of HA reaches a saturation limit between $\sim 1100-1300{ }^{\circ} \mathrm{C}$ with closed porosity [50].

Since the hydroxyapatite used was initially well crystallized, non-alteration of its crystallinity was observed. However, a decrease in the spectra intensity or resolution can be noticed after reaching $1250^{\circ} \mathrm{C}$. It was attributed to the loss of signal due to the movement in the z-axis of the sample during the in-situ measurement caused for the shrinkage of the part due to the pyrolysis of the resin and sintering.

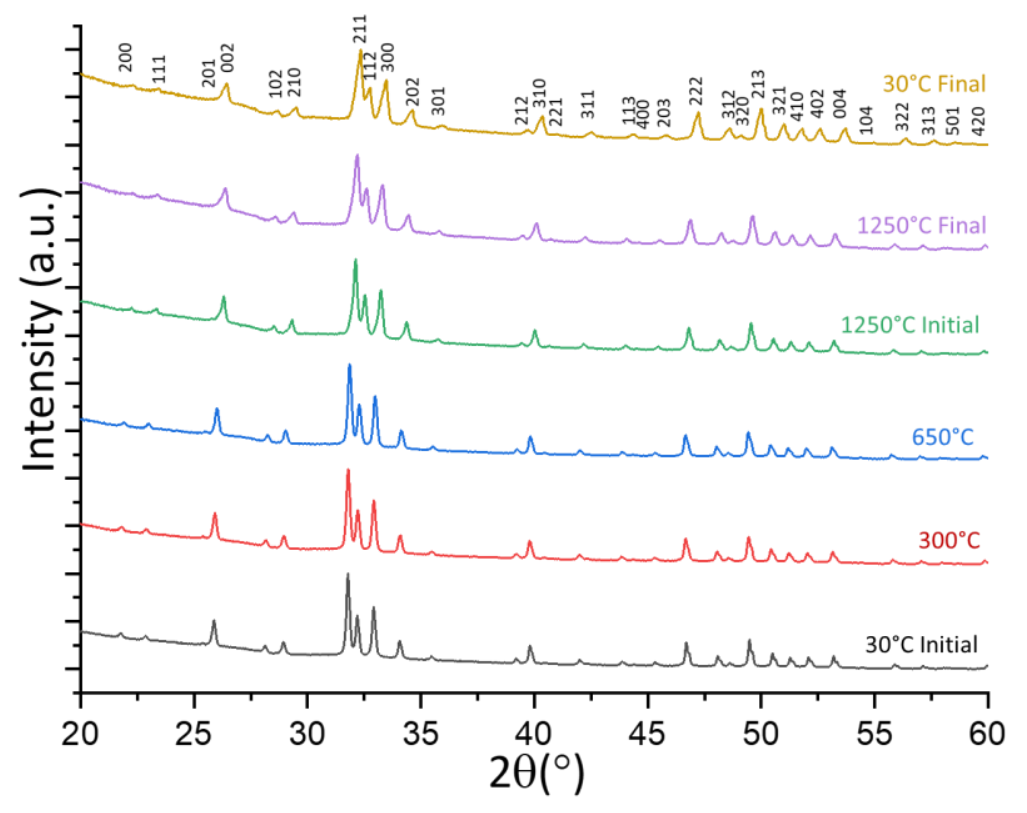

Figure 12. In-situ XRD diffractograms of the HA scaffolds during the debinding-sintering process at different temperatures. All the diffraction peaks correspond to stoichiometric hydroxyapatite (JCPDS 00009-0432).

Characterization of the cured resin with HA powder was performed by differential scanning calorimetry (DSC) and thermogravimetric analysis (TGA) to better understand the thermal treatments, especially the critical process of debinding. This characterization gives information on weight loss and involved energy during the debinding and sintering processes. The purpose of this study is to analyse the thermal degradation of the cured resin in the HA scaffold green body under air atmosphere. The TGA and DSC results for cured green body HA scaffold, optimized at 25 wt. \% (base resin + PEG200 diluent) and 75 wt. \% HAPBM powder 
is shown in Figure 13. The curve represents the weight loss and the energy involved during the thermal treatment, at a constant heating rate of $10^{\circ} \mathrm{C} \cdot \mathrm{min}^{-1}$ from room temperature until $1250^{\circ} \mathrm{C}$ in air atmosphere.

Three main weight loss stages can be distinguished related to the formation of volatiles, which diffuse from the interior of the part to the outside. These events represent the most critical temperature ranges during the debinding process since they indicate the escape of the gases generated and the probability of cracking of the green body.

- The first stage finishes at $240^{\circ} \mathrm{C}$ with a 3 wt. $\%$ of weight loss indicated by an exothermic peak, which was related to the loss of physically adsorbed water. At the same time, some organic matter such as acrylate resin or PEG200 experience soften, melt, and crack [25].

- The second stage is observed from $240^{\circ} \mathrm{C}$ to $498^{\circ} \mathrm{C}$ corresponding to the highest rate of weight loss, removing $22 \mathrm{wt} \%$ of the material. This was related to the thermal decomposition of the photocurable resin, including carbon-containing compounds [51]. Some organic components were burned and carbonized step by step.

- The third stage was defined as the thermal oxidation of the carbonized residue which does not substantially consume heat and has a minor influence on the TGA analysis [25]. When the temperature reached $1200^{\circ} \mathrm{C}$, the mass curve tended to be stable.

Two exothermic events were detected, associated with the three weight-loss events. Thus, indicating that the thermal degradation of the resin used for the suspensions releases energy during the thermal process. Indeed, the most intense exothermic peak is related to the weight loss event corresponding to the degradation of the carbon-containing compounds during the polymer degradation. On the whole, total organic removal is observed with a weight loss of $\sim 25 \mathrm{wt}$. \%. It is important to point out that the heating rates used during the debinding-sintering process of the scaffolds are between 10 and 5 times lower than the used for the TGA and DSC analyses. It is expected to produce deeper pyrolysis of the polymeric phase and at the same time control the diffusion of gases avoiding the formation of cracks. The results obtained for the thermal analyses are in correlation with the observed in literature for similar resin photocurable thermal degradation studies $[21,25,51]$. 


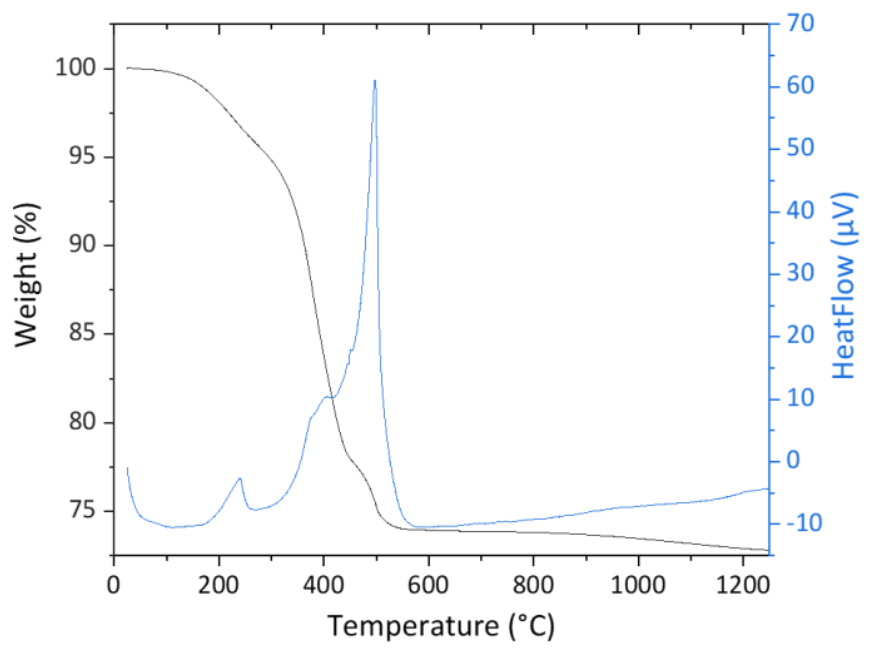

Figure 13. Differential scanning calorimetry (DSC) and thermogravimetric analysis (TGA) of HA green body scaffold.

A FTIR analysis was performed to confirm the compounds present in the parts after the whole production process. For comparison, the powder $\left(\mathrm{HA}_{\mathrm{PBM}}\right)$ used as filler during the preparation of the slurry was also analysed. Figure 14 shows the FTIR spectra of the starting powder and the HA scaffold produced. Small variations can be noticed between them. In both spectra the $\mathrm{PO}_{4}{ }^{3-}$ and structural $\mathrm{OH}^{-}$vibration bands corresponding to HA are clearly visible at $1092 \mathrm{~cm}^{-1}, 1040 \mathrm{~cm}^{-1}, 962 \mathrm{~cm}^{-1}, 601 \mathrm{~cm}^{-1}, 575 \mathrm{~cm}^{-1}, 561 \mathrm{~cm}^{-1}$, and at $650 \mathrm{~cm}^{-1}, 3600 \mathrm{~cm}^{-1}$ [47]. No secondary species were detected before or after the whole HA scaffold production process. 


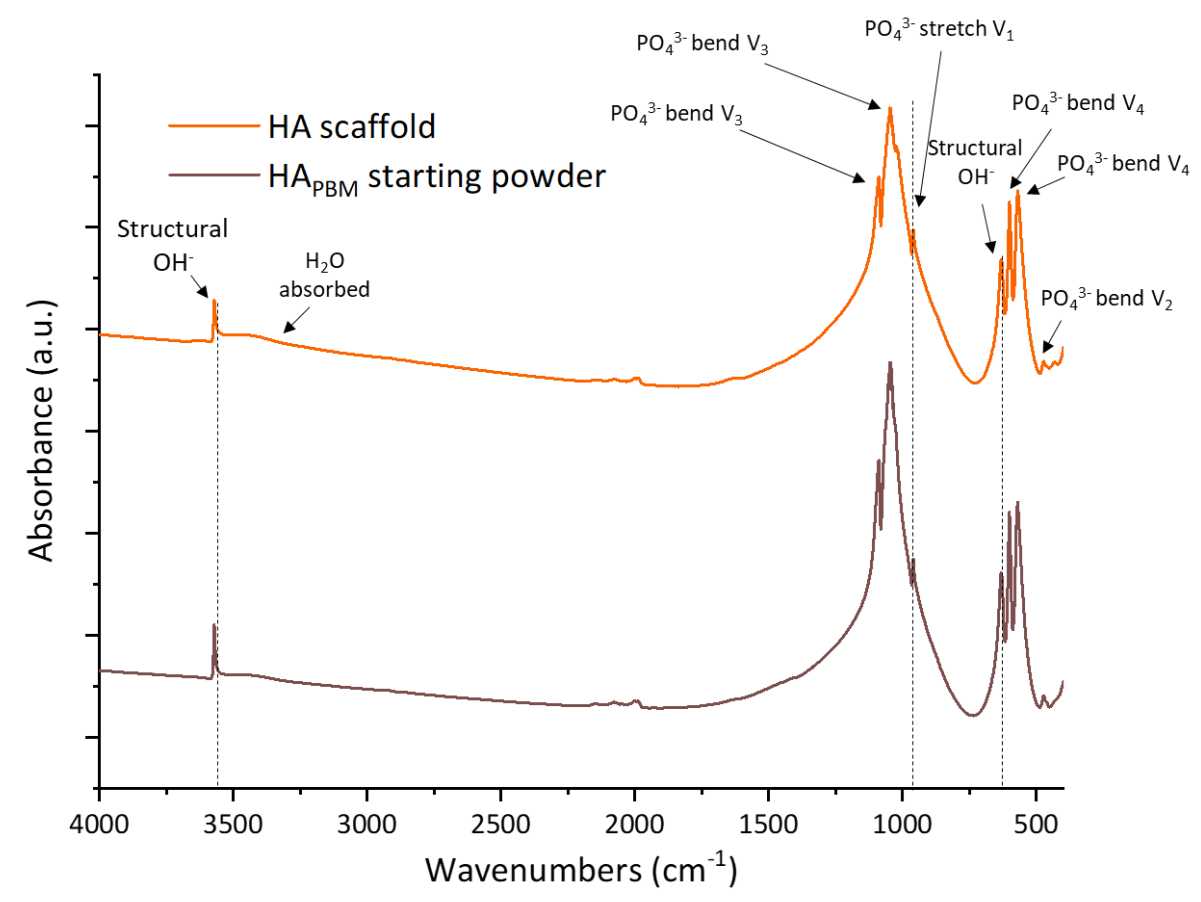

Figure 14. FTIR spectrum analysis of the HA before and after the SD process.

\subsubsection{Macro-structural properties and physical changes of sintered HA scaffolds}

HA bioceramic scaffolds were examined to evaluate their macro-structural properties (surface finishing, porosity, and density) and physical changes (shrinkage) after the debinding-sintering process. Figure 15 shows the photographs of the green body and sintered HA scaffolds produced by the MSLA process. Figure 15a shows the differences in dimension between the green body scaffold and its homologous after the debinding-sintering process due to the pyrolysis-induced shrinking. However, no fracture or crack was detected after the debinding-sintering process. Figure 15b shows differences between the dimensions of the scaffolds printed at different orientation angles (shrinkage values can be observed in Table 3). The differences in dimension were only observed once the parts were submitted to the debinding-sintering process. It indicates a physical anisotropic effect produced by the shrinkage which depends on the orientation in which the layers forming the part was printed. This kind of anisotropic physical change has been reported in previous studies on the 3D printing of ceramic parts [21,52]. 


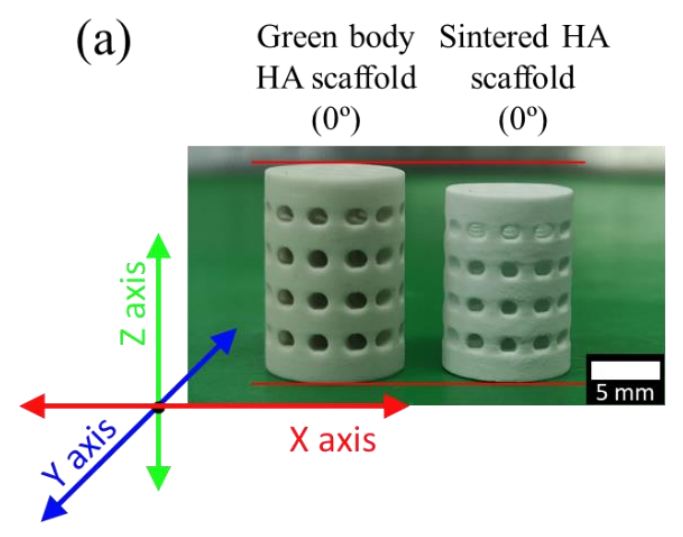

(b) Printing orientations

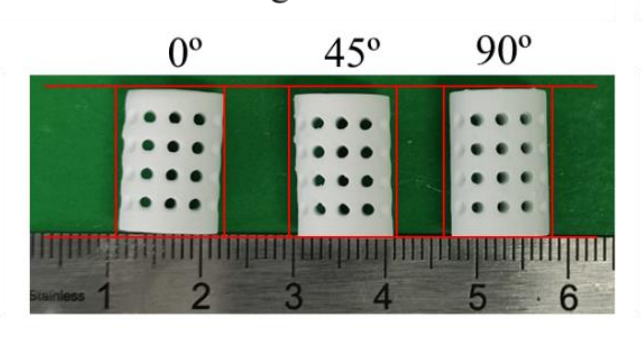

Figure 15. Visual examination of HA parts: (a) comparison between green body and sintered HA scaffolds (including axes arrows), (b) comparison between sintered HA scaffolds printed at three different orientations $\left(0^{\circ}, 45^{\circ}\right.$, and $\left.90^{\circ}\right)$.

Table 3 shows the general information related to the macro-structural properties of the produced HA scaffolds. The calculated average pyrolysis-induced shrinkage shows that there is a different effect depending on the axis. For parts printed at the same orientation, a higher distortion effect was measured for the axes perpendicular to the printing direction (e.g., z-axis for parts printed at $0^{\circ}$ or $\mathrm{x}-\mathrm{y}$ plane).

No big differences in the total porosity were observed between the three orientations. A higher porosity was obtained when using the $\mathrm{Hg}$ intrusion porosimetry method, this could indicate that the resolution of the CT analysis $(9 \mu \mathrm{m})$ was not enough to take into account the microporosity. The total porosity of the scaffolds is close to the common cancellous bone porosity of 50-90\% [53].

Almost similar relative densities were obtained for the three kinds of scaffolds $\left(2.84{\mathrm{~g} . \mathrm{cm}^{-3}}^{-}\right)$ demonstrating a good capability of the MSLA technique in the reproduction of HA scaffolds with an accurate controlled and interconnected macroporosity. The obtained density is lower than the hydroxyapatite density $\left(3.16 \mathrm{~g} . \mathrm{cm}^{-3}\right)$, thus corresponding to the expected values for a scaffold with open and close porosity. 
Table 3. Average shrinkage in $x-y$ and $z$-axis of parts after the debinding-sintering process for each of the three printing orientations. Average porosity and relative densities of sintered HA scaffolds determined by different methods. $(N=10)$

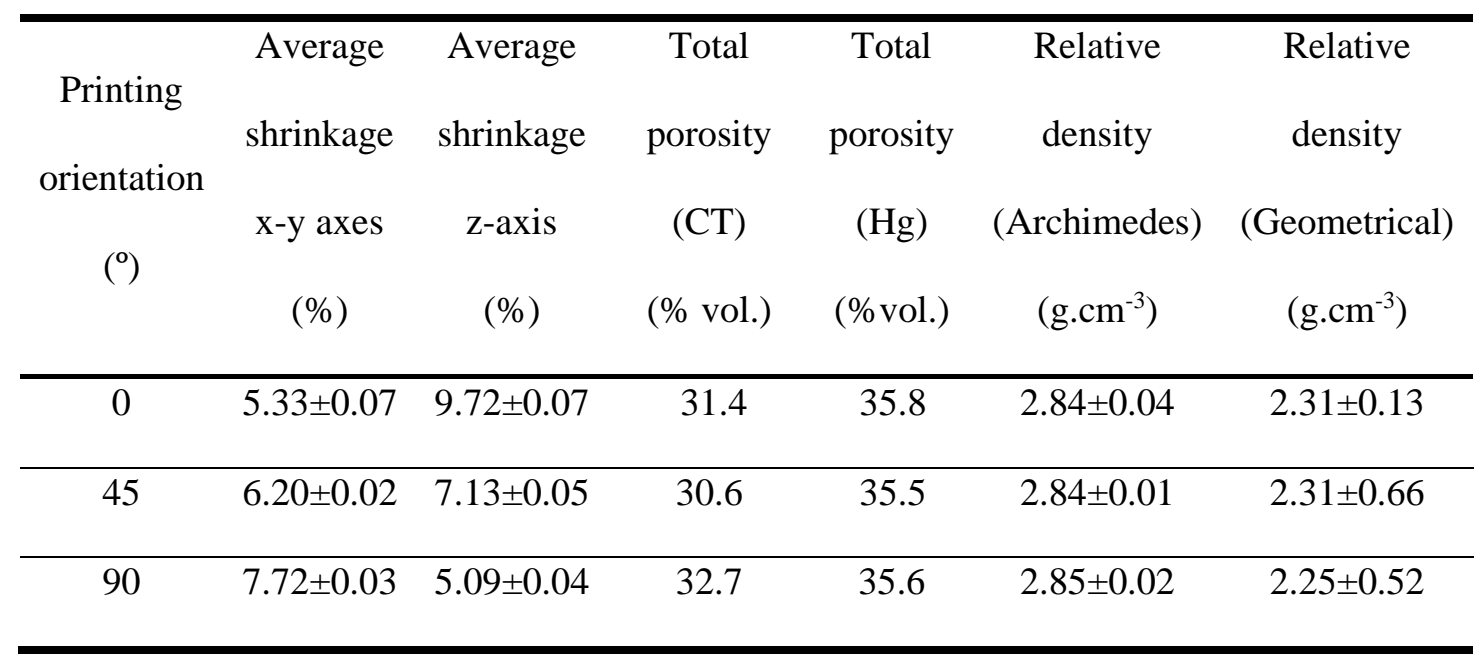

The SEM micrographs of the HA scaffolds surface printed at three different orientations are shown in Figure 16. SEM micrographs were taken at a similar X-Z plane (front view) of the parts for comparison. The macroporosity of the scaffolds is composed of an interconnected porosity with a pore size of about $1000 \mu \mathrm{m}$. This result fulfils the objective of producing scaffolds with controlled macroporosity (pore size $>400 \mu \mathrm{m}$ ) which could promote the attachment, growth, and proliferation of bone cells inside of the scaffold enhancing the new bone formation and the formation of capillaries. This macro-porosity should then provide transport pathways for oxygen, wastes, and nutrients necessary to maintain living cells [54,55]. Different surface finishing is observed in this plane depending on the printing orientation with a clear view of the layers for the scaffolds printed at $0^{\circ}$ and $45^{\circ}$. However, no significant pores in the microstructures can be observed due to any delamination or imperfect bonding between layers. This indicates a good sinterability of the HA powder. 


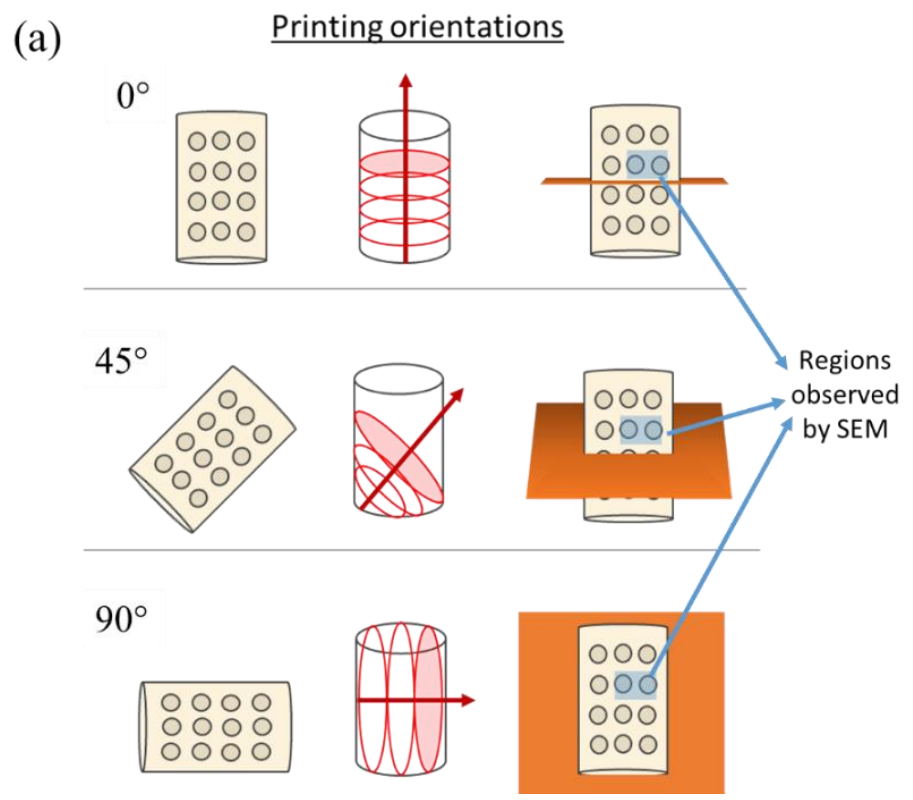

(b)
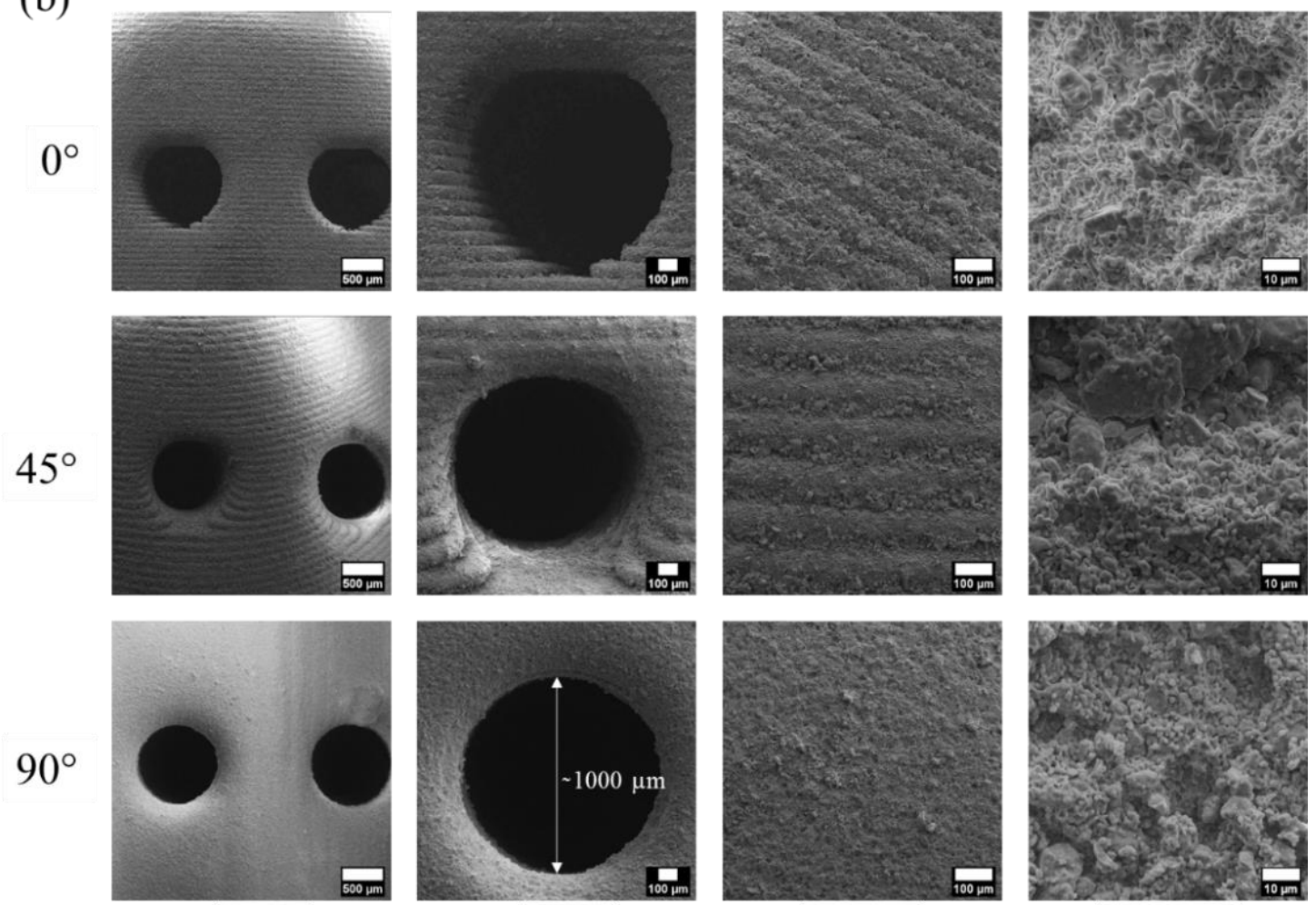

$20 \mathrm{x}$

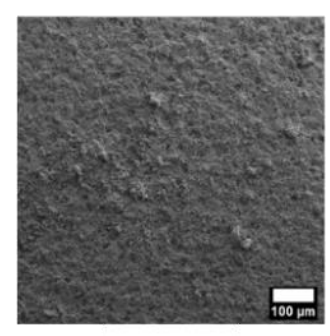

$100 x$

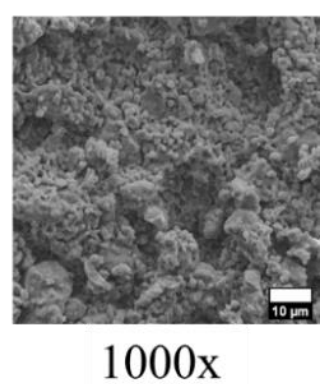

Figure 16. (a) Three different schematic representations of the three printing orientations employed. (b) SEM micrographs at different magnifications of the $H A$ scaffolds ( $X, Z$ plane) printed at three different orientations. 
Additionally, to analyse the internal microstructure of the HA scaffolds the cross-section at the X-Y plane (top view) was observed by SEM. These micrographs are shown in Figure 17. From a general view, no induced anisotropy was identified due to the printing process on the three types of scaffolds. The three samples presented micropores of about 10-15 $\mu \mathrm{m}$ of diameter corresponding to the microstructural porosity of the sintered HA. This porosity could allow the migration and proliferation of osteoblasts $(15-50 \mu \mathrm{m})$, and especially stem cells $(5-12 \mu \mathrm{m})$. The HA crystal sizes correspond with the particle size of the HA powder used for the preparation of the scaffolds. A dispersed distribution of HA grains was observed with sizes from $2.5 \mu \mathrm{m}$ to $20 \mu \mathrm{m}$ of diameter.

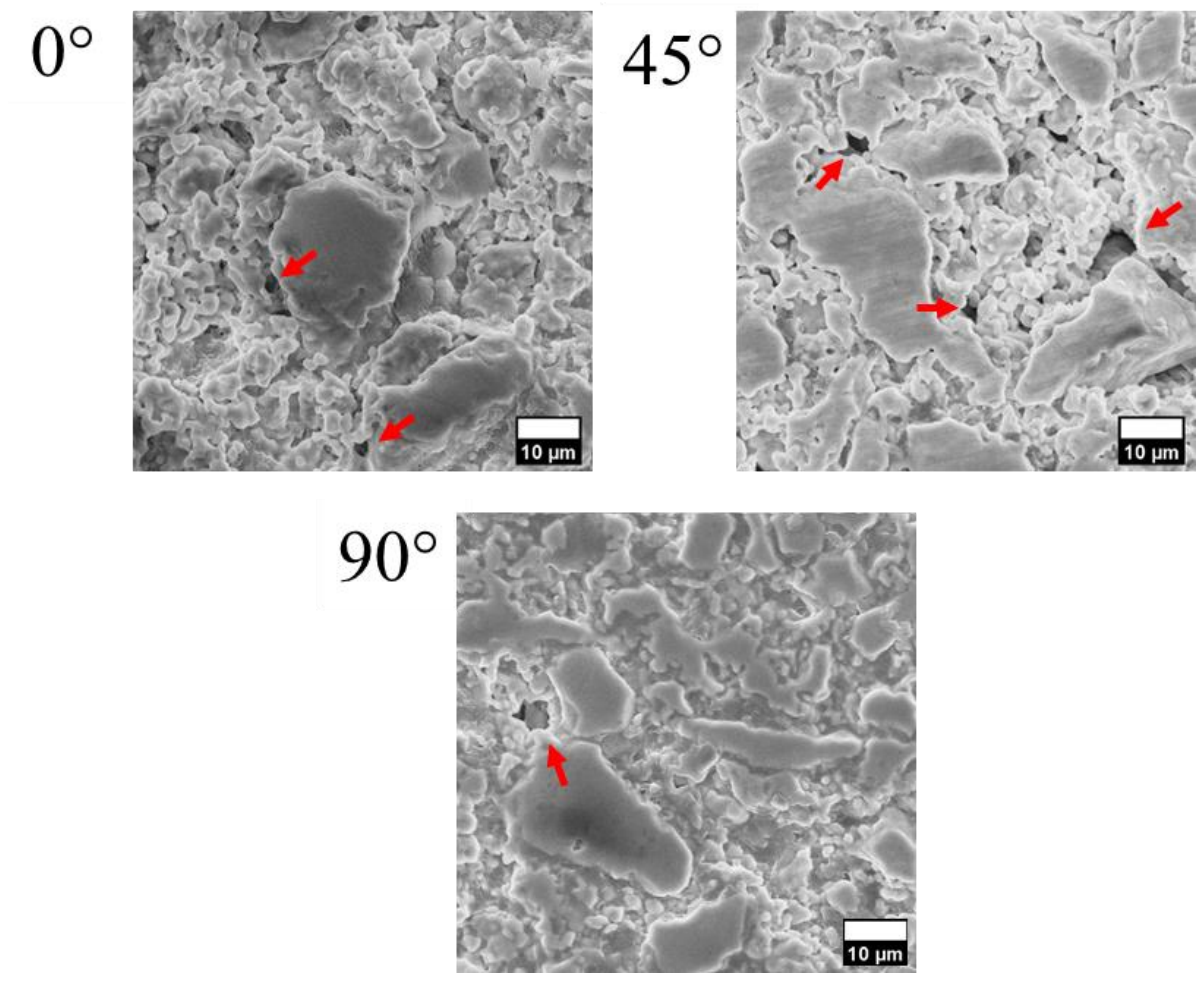

Figure 17. Cross-section of parts of the HA scaffolds (X, Y plane) printed at three different orientation degrees. Micropores are remarked by red arrows.

Three-dimensional reconstructions of the sintered scaffolds using X-ray micro-computed tomography are also illustrated in Figure 18. A defect volume scale was added for a better comparison of the pore volume. The three samples showed accurate controlled and interconnected macroporosity with a total 
volume in the range of $300-350 \mathrm{~mm}^{3}$. The cross-section views of HA scaffolds in X-Y and Y-Z planes did not show any kind of anisotropy induced by the MSLA process. This homogeneity observed for the whole volume of the parts indicates suitability for the processing of suspensions by MSLA technique on the production of HA scaffolds with controlled macroporosity. Additional CT results showing the internally interconnected porosity of the scaffold printed at $0^{\circ}$ can be found in Figure $\mathrm{S} 1$.

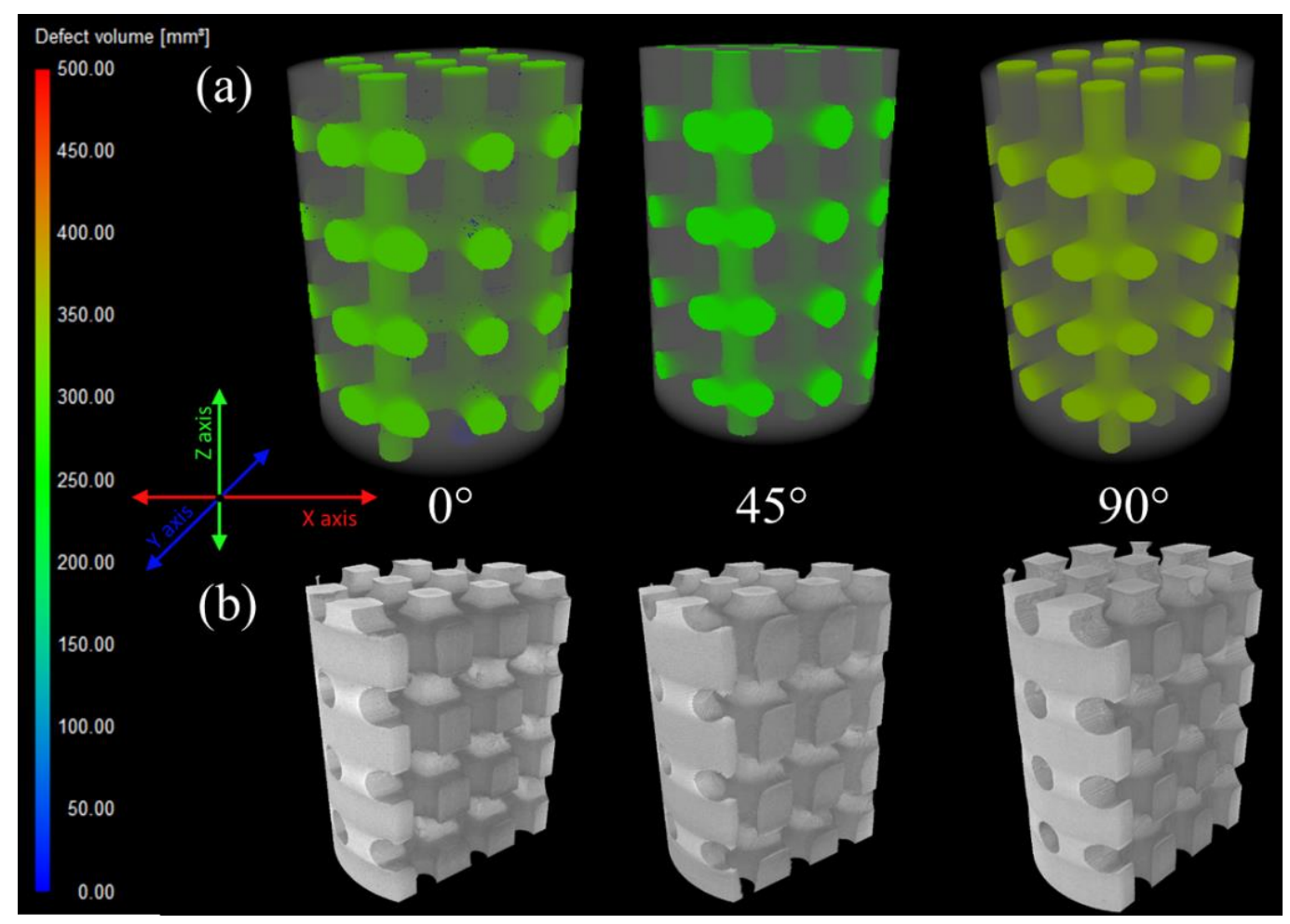

Figure 18. Visualization obtained using X-ray micro-computed tomography of the three kinds of HA scaffolds $\left(0^{\circ}, 45^{\circ}\right.$, and $\left.90^{\circ}\right)$ produced by MSLA after the debinding-sintering treatment. (a) The pore distribution of HA scaffolds with previously modelled interconnected macroporosity and circular overtures of $1000 \mu \mathrm{m}$ in the three directions. (b) Cross-section views of HA scaffolds in $X-Y$, and $Y-Z$ planes. 


\subsubsection{Mechanical properties}

The anisotropic effect of the printing orientation on the scaffolds mechanical properties was examined. Table 4 shows the compression strength of the sintered scaffolds as a function of the orientation in which they were printed. Mechanical strength is primarily controlled by pore volume and distribution, however, in this case, the total porosity of the scaffolds has been demonstrated to not be affected by the printing orientation. Thus, the total pore volume of the samples studied was almost similar (Table 3). Scaffolds with $0^{\circ}$ orientation are weakest in compressive strength $(4.3 \pm 0.4 \mathrm{MPa})$ since the plane in which they are printed is perpendicular to the direction of load. With the inclination of the plane, it becomes diagonal to the loading

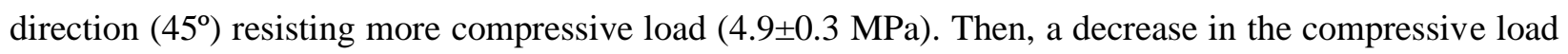
is observed for $\left(90^{\circ}\right)$ as the printing orientation is parallel to the direction of loading $(4.8 \pm 0.2 \mathrm{MPa})$. These results are in line with Lee et al. [56] and Saini et al. [57] which said that the diagonally printed specimens $\left(45^{\circ}\right)$ have higher compressive strength as compared to axial and transverse 3D printed parts. Indeed, they are in accordance with the results obtained by Feng et al. [21] whose HA printed scaffolds (at orientation $0^{\circ}$ and using a 50 vol. $\%$ suspension) with higher porosity than the ones produced in the present study showed lower compressive strength. The values obtained for the three types of scaffolds are found to be within the range of values reported for cancellous bone compressive strength (1-12 MPa) for porosity between 50 and $90 \%$. Then, to advance this study, the porosity of the scaffolds $(\sim 31 \%)$ could be increased to reproduce closer conditions [53].

Table 4. Compressive strength of HA scaffolds depending on the printing orientation. $(N=10)$

\begin{tabular}{cc}
\hline Printing orientation $\left(^{\circ}\right)$ & Compression strength (MPa) \\
\hline 0 & $4.2 \pm 0.4$ \\
\hline 45 & $4.9 \pm 0.3$ \\
\hline 90 & $4.8 \pm 0.2$ \\
\hline
\end{tabular}


The compressive stress-strain curves obtained for the bioceramic HA scaffolds printed at $45^{\circ}$ are shown in Figure S2a. These curves are characterized by an initial non-linear toe region (first strain stage) followed by a main linear region till the failure point. The inexistence of a concave shape before the failure point and the sharp peaks are common in brittle materials as ceramics. It is known that brittle materials, when subjected to stress, break with little elastic deformation and without significant plastic deformation. For this reason, brittle materials absorb relatively little energy before the fracture, even those of high strength [58]. Cracks propagate rapidly along the compression loading direction as shown in Figure S2b. The fluctuations (or serration) in the compression stress-strain response are essentially attributed to the spalling of a small specimen volume, as the growing cracks either meet the free/unconstrained surface of the material or, coalesce between them [59]. This is in accordance with other works in the literature [21,52]. Brittle fracture with a transgranular cleavage is observed (cracks grow through the material grains) and no apparent plastic deformation takes place before fracture. No preferred direction was observed for the fracture as an effect of the orientation in which the specimens were printed.

\subsubsection{Dissolution rate}

The in vitro behaviour of the sintered HA scaffolds was preliminarily evaluated in terms of dissolution rate, $\mathrm{Ca}^{2+}$ ion release, and $\mathrm{pH}$ change of the dissolution medium $\left(0.05 \mathrm{M}\right.$ TRIS buffer solution) at $37 \pm 1^{\circ} \mathrm{C}$. The $\mathrm{pH}$ value evolution of the TRIS buffer solution is shown in Figure 19a. The initial pH of the TRIS buffer solution was 7.3. Then, this value was decreasing with the increase of the HA scaffold soaking time. After $72 \mathrm{~h}$ the $\mathrm{pH}$ reached a value of 7.24. The $\mathrm{pH}$ variation surrounding an implant is an indicator of the in vivo degradation. A fast unintentional degradation in contact with the environment can deteriorate the mechanical properties of the implant [60]. In this case, a low variation of the $\mathrm{pH}$ was observed (lower than \pm 0.3 ) indicating a low degradation of the HA scaffold. The scaffold weight loss and $\mathrm{Ca}^{2+}$ ion concentrations in the solution at different soaking times are given in Figure 19b. A slight weight loss of the HA scaffolds was observed after $24 \mathrm{~h}$. It increased with the soaking time until a $0.12 \%$ of loss of the total scaffold mass 
at $72 \mathrm{~h}$. In addition, a low $\mathrm{Ca}^{2+}$ ion release $\left(1.26 \mathrm{mg} \cdot \mathrm{l}^{-1}\right)$ was observed during the time of the measurement. These results were attributed to the high crystallinity of the HA phase (due to the sintering process) which is known to decrease the dissolution rate [61].

It is known that depending on the calcium phosphate phase the dissolution rate varies, being HA one of the phases showing low rates [62]. Some phases like TCP may dissolve fast and replace the coating or cement with bone. This property depends on the calcium phosphate phase content of the scaffold, crystallinity, particle size, and porosity. A higher degree of porosity means a higher surface area in contact between the implant and the body fluids, inducing a faster dissolution rate. Then, a higher degree of crystallinity of the calcium phosphate structure can reduce the solubility of the scaffolds, thus explaining the large differences in solubility between scaffolds prepared with the same calcium phosphate phase $[61,63]$. The rate of bone substitution also depends on the age, sex, and general metabolic health of the recipient, even on the anatomic site, thus the desired resorption rate is the rate comparable to the formation of bone tissue (between a few months and a few years), in the case of calcium phosphate it may take 3 to 36 months to be replaced by bone [64].

(a)

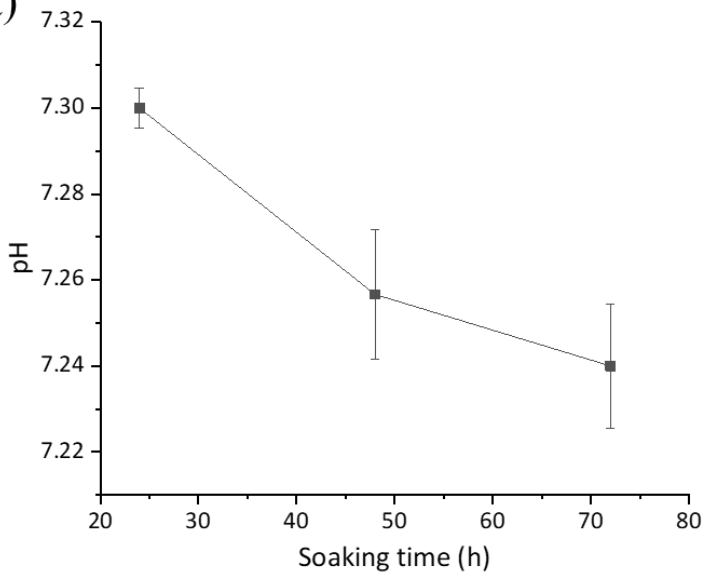

(b)

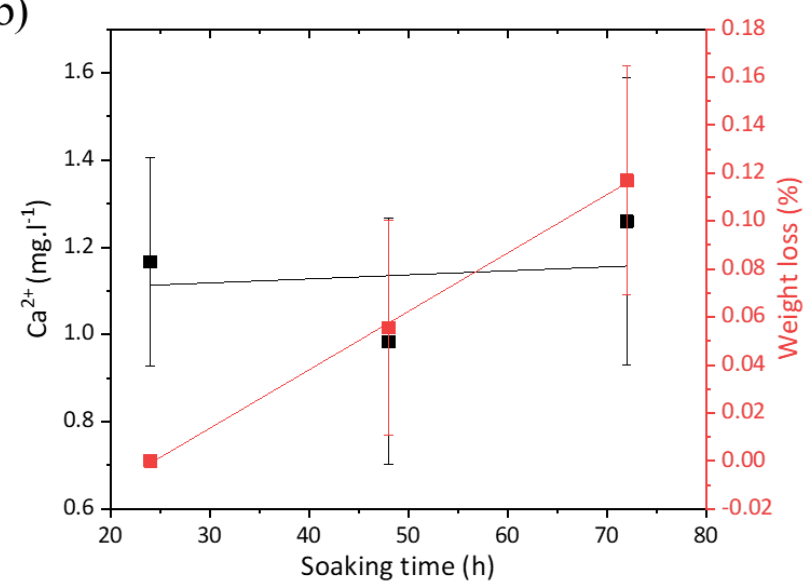

Figure 19. (a) TRIS solution $\mathrm{pH}$ evolution, (b) $\mathrm{Ca}^{2+}$ ion release, and scaffold weight loss evolution as a function of the sintered HA scaffold soaking time. $(N=3)$

\section{Conclusions}


In the present work, HA scaffolds were obtained by MSLA innovative AM process. HA powders with different characteristics were produced and employed for the preparation of HA-filled photocurable suspensions. The influence of the different powders on the properties of suspensions was evaluated. After a comparison of the rheological behaviour and dispersion stability of the suspensions, it was found that the powder tailored through the planetary ball mill process having an intermedium particle size showed the most outstanding results (viscosity $<5$ Pa.s at a shear rate $1 \mathrm{~s}^{-1}$ and TSI $<2$ for the duration of the process) at $50 \mathrm{vol} . \%$. The processability of suspensions was tested in a commercial MSLA apparatus through the production of scaffolds with controlled macroporosity. The process showed high accuracy in producing the macroporosity (pore size of $1000 \mu \mathrm{m}$ ) at three printing orientations $\left(0^{\circ}, 45^{\circ}\right.$, and $\left.90^{\circ}\right)$. A debinding-sintering process at $1250^{\circ} \mathrm{C}$ was employed for the complete pyrolysis of the resin and sintering of the scaffold. The chemical composition and macro-structural properties of the scaffolds were examined. It was observed that the debinding-sintering shrinkage of the parts was dependent on the printing orientation, with a higher shrinkage in the direction perpendicular to the printed plane. The analysis of the mechanical properties also showed a slight anisotropic effect induced by the printing orientation with the highest compressive strength $(4.9 \pm 0.3 \mathrm{MPa})$ obtained for the scaffolds printed diagonally $\left(45^{\circ}\right)$. Then, the dissolution rate test of the scaffolds showed a low variation in the TRIS solution $\mathrm{pH}$ with the soaking time and a slow dissolution rate, which was attributed to the high crystallinity and intrinsic stability of the HA calcium phosphate phase. In conclusion, the tailoring of HA powder suitable for its use as a filler in photocurable suspensions was confirmed. The HA specimens produced showed promising results for their use in surgery for guided bone regeneration in the case of minor or non-load bearing voids requiring slow resorption properties.

\section{Acknowledgements}

This project has received funding from the European Union's Horizon 2020 research and innovation programme under the Marie Skłodowska-Curie grant agreement No 764935.

\section{Data availability}


The raw/processed data required to reproduce these findings cannot be shared at this time as the data also forms part of an ongoing study.

\section{REFERENCES}

[1] Ageing Europe - statistics on population developments, (2020). https://ec.europa.eu/eurostat/statistics-explained/index.php?title=Ageing_Europe__statistics_on_population_developments.

[2] Office of the Surgeon General (US), Bone Health and Osteoporosis: A Report of the Surgeon General, Office of the Surgeon General (US), Rockville (MD), 2004. http://www.ncbi.nlm.nih.gov/books/NBK45513/.

[3] I. Benjumeda Wijnhoven, R. Vallejos, J.F. Santibanez, C. Millán, J.F. Vivanco, Analysis of cellbiomaterial interaction through cellular bridge formation in the interface between hGMSCs and CaP bioceramics, Sci Rep. 10 (2020) 16493. https://doi.org/10.1038/s41598-020-73428-y.

[4] D.W. Hutmacher, Scaffolds in tissue engineering bone and cartilage, Biomaterials. 21 (2000) 25292543. https://doi.org/10.1016/S0142-9612(00)00121-6.

[5] L. Zhang, Y. Morsi, Y. Wang, Y. Li, S. Ramakrishna, Review scaffold design and stem cells for tooth regeneration, Japanese Dental Science Review. $49 \quad$ (2013) 14-26. https://doi.org/10.1016/j.jdsr.2012.09.001.

[6] H. Schliephake, F.W. Neukam, Bone replacement with porous hydroxyapatite blocks and titanium screw implants: An experimental study, Journal of Oral and Maxillofacial Surgery. 49 (1991) 151156. https://doi.org/10.1016/0278-2391(91)90103-S.

[7] B.A. Gultekin, E. Bedeloglu, T.E. Kose, E. Mijiritsky, Comparison of Bone Resorption Rates after Intraoral Block Bone and Guided Bone Regeneration Augmentation for the Reconstruction of Horizontally Deficient Maxillary Alveolar Ridges, BioMed Research International. 2016 (2016) e4987437. https://doi.org/10.1155/2016/4987437. 
[8] M. Tumedei, P. Savadori, M. Del Fabbro, Synthetic Blocks for Bone Regeneration: A Systematic Review and Meta-Analysis, Int J Mol Sci. 20 (2019) 4221. https://doi.org/10.3390/ijms20174221.

[9] A. Monje, H.-L. Wang, Biological and physical properties of bone block grafting biomaterials for alveolar ridge augmentation, (n.d.) 14.

[10] J.T. Mellonig, Bone allografts in periodontal therapy, Clin Orthop Relat Res. (1996) 116-125. https://doi.org/10.1097/00003086-199603000-00014.

[11] E. Pamula, E. Filová, L. Bačáková, V. Lisá, D. Adamczyk, Resorbable polymeric scaffolds for bone tissue engineering: The influence of their microstructure on the growth of human osteoblast-like MG 63 cells, J. Biomed. Mater. Res. 89A (2009) 432-443. https://doi.org/10.1002/jbm.a.31977.

[12] A. Kurella, N.B. Dahotre, Review paper: Surface Modification for Bioimplants: The Role of Laser Surface Engineering, J Biomater Appl. 20 (2005) 5-50. https://doi.org/10.1177/0885328205052974.

[13] H. Qu, Additive manufacturing for bone tissue engineering scaffolds, Materials Today Communications. 24 (2020) 101024. https://doi.org/10.1016/j.mtcomm.2020.101024.

[14] H.H. Yen, P.G. Stathopoulou, CAD/CAM and 3D-printing Applications for Alveolar Ridge Augmentation, Curr Oral Health Rep. 5 (2018) 127-132. https://doi.org/10.1007/s40496-018-01804.

[15] L.C. Hwa, S. Rajoo, A.M. Noor, N. Ahmad, M.B. Uday, Recent advances in 3D printing of porous ceramics: A review, Current Opinion in Solid State and Materials Science. 21 (2017) 323-347. https://doi.org/10.1016/j.cossms.2017.08.002.

[16] K. Lin, R. Sheikh, S. Romanazzo, I. Roohani, 3D Printing of Bioceramic Scaffolds-Barriers to the Clinical Translation: From Promise to Reality, and Future Perspectives, Materials. 12 (2019) 2660. https://doi.org/10.3390/ma12172660.

[17] S. Zakeri, M. Vippola, E. Levänen, A comprehensive review of the photopolymerization of ceramic resins used in stereolithography, Additive Manufacturing. $35 \quad$ (2020) 101177. https://doi.org/10.1016/j.addma.2020.101177. 
[18] J.R. Zyzalo, Masked Projection Stereolithography: Improvement of the Limaye Model for Curing Single Layer Medium Sized Parts, (2008) 274.

[19] H. Quan, T. Zhang, H. Xu, S. Luo, J. Nie, X. Zhu, Photo-curing 3D printing technique and its challenges, Bioactive Materials. 5 (2020) 110-115. https://doi.org/10.1016/j.bioactmat.2019.12.003.

[20] X. Li, Y. Yuan, L. Liu, Y.-S. Leung, Y. Chen, Y. Guo, Y. Chai, Y. Chen, 3D printing of hydroxyapatite/tricalcium phosphate scaffold with hierarchical porous structure for bone regeneration, Bio-Des. Manuf. 3 (2020) 15-29. https://doi.org/10.1007/s42242-019-00056-5.

[21] C. Feng, K. Zhang, R. He, G. Ding, M. Xia, X. Jin, C. Xie, Additive manufacturing of hydroxyapatite bioceramic scaffolds: Dispersion, digital light processing, sintering, mechanical properties, and biocompatibility, J Adv Ceram. 9 (2020) 360-373. https://doi.org/10.1007/s40145-020-0375-8.

[22] K. Zhang, C. Xie, G. Wang, R. He, G. Ding, M. Wang, D. Dai, D. Fang, High solid loading, low viscosity photosensitive A12O3 slurry for stereolithography based additive manufacturing, Ceramics International. 45 (2019) 203-208. https://doi.org/10.1016/j.ceramint.2018.09.152.

[23] S.Y. Song, M.S. Park, D. Lee, J.W. Lee, J.S. Yun, Optimization and characterization of high-viscosity $\mathrm{ZrO} 2$ ceramic nanocomposite resins for supportless stereolithography, Materials \& Design. 180 (2019) 107960. https://doi.org/10.1016/j.matdes.2019.107960.

[24] M. Pfaffinger, G. Mitteramskogler, R. Gmeiner, J. Stampfl, Thermal Debinding of Ceramic-Filled Photopolymers, MSF. 825-826 (2015) 75-81. https://doi.org/10.4028/www.scientific.net/MSF.825826.75 .

[25] Q. Chen, B. Zou, Q. Lai, Y. Wang, R. Xue, H. Xing, X. Fu, C. Huang, P. Yao, A study on biosafety of HAP ceramic prepared by SLA-3D printing technology directly, Journal of the Mechanical Behavior of Biomedical Materials. 98 (2019) 327-335. https://doi.org/10.1016/j.jmbbm.2019.06.031.

[26] F.P.W. Melchels, J. Feijen, D.W. Grijpma, A review on stereolithography and its applications in biomedical engineering, $\quad$ Biomaterials. $\quad 31 \quad$ (2010) $\quad$ 6121-6130. https://doi.org/10.1016/j.biomaterials.2010.04.050. 
[27] H. Li, Y. Liu, Y. Liu, K. Hu, Z. Lu, J. Liang, Effects of Solvent Debinding on the Microstructure and Properties of 3D-Printed Alumina Ceramics, ACS Omega. 5 (2020) 27455-27462. https://doi.org/10.1021/acsomega.0c03944.

[28] M. Tourbin, F. Brouillet, B. Galey, N. Rouquet, P. Gras, N. Abi Chebel, D. Grossin, C. Frances, Agglomeration of stoichiometric hydroxyapatite: Impact on particle size distribution and purity in the precipitation and maturation steps, Powder Technology. $360 \quad$ (2020) 977-988. https://doi.org/10.1016/j.powtec.2019.10.050.

[29] M.A. Inam, S. Ouattara, C. Frances, Effects of concentration of dispersions on particle sizing during production of fine particles in wet grinding process, Powder Technology. 208 (2011) 329-336. https://doi.org/10.1016/j.powtec.2010.08.025.

[30] P. Navarrete-Segado, M. Tourbin, D. Grossin, C. Frances, Tailoring hydroxyapatite suspensions by stirred bead milling as pre-processing step for additive manufacturing technologies., Adv. Powder Technol. (Under Review). (2021).

[31] Phrozen Shuffle \& Shuffle XL User Manuals, Dropbox Paper. (n.d.). https://phrozen3d.com/pages/manual.

[32] V. Raikos, Encapsulation of vitamin E in edible orange oil-in-water emulsion beverages: Influence of heating temperature on physicochemical stability during chilled storage, Food Hydrocolloids. 72 (2017) 155-162. https://doi.org/10.1016/j.foodhyd.2017.05.027.

[33] C.A. Schneider, W.S. Rasband, K.W. Eliceiri, NIH Image to ImageJ: 25 years of image analysis, Nat Methods. 9 (2012) 671-675. https://doi.org/10.1038/nmeth.2089.

[34] F. Granados-Correa, J. Bonifacio-Martínez, J. Serrano-Gómez, The ball milling effect on tribasic calcium phosphate and its chromium (VI) ion sorption properties, Journal of The Chilean Chemical Society - J CHIL CHEM SOC. 54 (2009). https://doi.org/10.4067/S0717-97072009000300010.

[35] P.L. Guzzo, J.B. Santos, R.C. David, Particle size distribution and structural changes in limestone ground in planetary ball mill, International Journal of Mineral Processing. 126 (2014) 41-48. https://doi.org/10.1016/j.minpro.2013.11.005. 
[36] R. Newburgh, J. Peidle, W. Rueckner, Einstein, Perrin, and the reality of atoms: 1905 revisited, American Journal of Physics - AMER J PHYS. 74 (2006). https://doi.org/10.1119/1.2188962.

[37] D. Komissarenko, P. Sokolov, A. Evstigneeva, I. Shmeleva, A. Dosovitsky, Rheological and Curing Behavior of Acrylate-Based Suspensions for the DLP 3D Printing of Complex Zirconia Parts, Materials. 11 (2018) 2350. https://doi.org/10.3390/ma11122350.

[38] M. Nutan, I. Reddy, General Principles of Suspensions, in: Pharmaceutical Suspensions: From Formulation Development to Manufacturing, 2009: pp. 39-65. https://doi.org/10.1007/978-1-44191087-5_2.

[39] D. Quemada, Rheological modelling of complex fluids. I. The concept of effective volume fraction revisited, Eur. Phys. J. AP. 1 (1998) 119-127. https://doi.org/10.1051/epjap:1998125.

[40] J.J. Stickel, R.L. Powell, Fluid Mechanics and Rheology of Dense Suspensions, Annual Review of Fluid Mechanics. 37 (2005) 129-149. https://doi.org/10.1146/annurev.fluid.36.050802.122132.

[41] I.M. Krieger, T.J. Dougherty, A Mechanism for Non-Newtonian Flow in Suspensions of Rigid Spheres, Transactions of the Society of Rheology. 3 (1959) 137-152. https://doi.org/10.1122/1.548848.

[42] V. Tomeckova, J.W. Halloran, Flow behavior of polymerizable ceramic suspensions as function of ceramic volume fraction and temperature, Journal of the European Ceramic Society. 31 (2011) 25352542. https://doi.org/10.1016/j.jeurceramsoc.2011.01.019.

[43] T.-M.G. Chu, J.W. Halloran, High-Temperature Flow Behavior of Ceramic Suspensions, Journal of the American Ceramic Society. 83 (2004) 2189-2195. https://doi.org/10.1111/j.11512916.2000.tb01534.x.

[44] T.E. Baldock, M.R. Tomkins, P. Nielsen, M.G. Hughes, Settling velocity of sediments at high concentrations, $\quad$ Coastal $\quad$ Engineering. $\quad 51 \quad 91-100$. https://doi.org/10.1016/j.coastaleng.2003.12.004. 
[45] N.-S. Cheng, Effect of Concentration on Settling Velocity of Sediment Particles, Journal of Hydraulic Engineering-ASCE. $123 \quad$ (1997) 728-731. https://doi.org/10.1061/(ASCE)07339429(1997)123:8(728).

[46] J.F. Richardson, W.N. Zaki, Sedimentation and fluidisation. Part 1., Trans. Inst. Chem. Eng. 32 (1954) $35-53$.

[47] C. Rey, O. Marsan, C. Combes, C. Drouet, D. Grossin, S. Sarda, Characterization of Calcium Phosphates Using Vibrational Spectroscopies, in: B. Ben-Nissan (Ed.), Advances in Calcium Phosphate Biomaterials, Springer Berlin Heidelberg, Berlin, Heidelberg, 2014: pp. 229-266. https://doi.org/10.1007/978-3-642-53980-0_8.

[48] K. Tõnsuaadu, K.A. Gross, L. Plūduma, M. Veiderma, A review on the thermal stability of calcium apatites, J Therm Anal Calorim. 110 (2012) 647-659. https://doi.org/10.1007/s10973-011-1877-y.

[49] S. Ramesh, K.L. Aw, R. Tolouei, M. Amiriyan, C.Y. Tan, M. Hamdi, J. Purbolaksono, M.A. Hassan, W.D. Teng, Sintering properties of hydroxyapatite powders prepared using different methods, Ceramics International. 39 (2013) 111-119. https://doi.org/10.1016/j.ceramint.2012.05.103.

[50] M. Prakasam, J. Locs, K. Salma-Ancane, D. Loca, A. Largeteau, L. Berzina-Cimdina, Fabrication, Properties and Applications of Dense Hydroxyapatite: A Review, J Funct Biomater. 6 (2015) 10991140. https://doi.org/10.3390/jfb6041099.

[51] J.G. Fernandes, Development and optimization of a Low Temperature Co-fired Ceramic suspension for Mask-Image-Projection-based Stereolithography, Ph.D. Thesis, Universitat de Barcelona, 2019. http://www.tdx.cat/handle/10803/667478.

[52] A. Farzadi, M. Solati-Hashjin, M. Asadi-Eydivand, N.A.A. Osman, Effect of Layer Thickness and Printing Orientation on Mechanical Properties and Dimensional Accuracy of 3D Printed Porous Samples for Bone Tissue Engineering, PLOS ONE. 9 (2014) e108252. https://doi.org/10.1371/journal.pone.0108252.

[53] T.-R. Kim, M.-S. Kim, T.S. Goh, J.S. Lee, Y.H. Kim, S.-Y. Yoon, C.-S. Lee, Evaluation of Structural and Mechanical Properties of Porous Artificial Bone Scaffolds Fabricated via Advanced TBA-Based 
Freeze-Gel Casting Technique, Applied $\quad$ Sciences. $9 \quad$ (2019) 1965. https://doi.org/10.3390/app9091965.

[54] V. Karageorgiou, D. Kaplan, Porosity of 3D biomaterial scaffolds and osteogenesis, Biomaterials. 26 (2005) 5474-5491. https://doi.org/10.1016/j.biomaterials.2005.02.002.

[55] M. Houmard, Q. Fu, M. Genet, E. Saiz, A.P. Tomsia, On the structural, mechanical, and biodegradation properties of $\mathrm{HA} / \beta-\mathrm{TCP}$ robocast scaffolds, Journal of Biomedical Materials Research. 101 (2013) 9. https://doi.org/10.1002/jbm.b.32935.

[56] C.S. Lee, S.G. Kim, H.J. Kim, S.H. Ahn, Measurement of anisotropic compressive strength of rapid prototyping parts, Journal of Materials Processing Technology. 187-188 (2007) 627-630. https://doi.org/10.1016/j.jmatprotec.2006.11.095.

[57] J. Saini, L. Dowling, J. Kennedy, D. Trimble, Investigations of the mechanical properties on different print orientations in SLA 3D printed resin, Proceedings of the Institution of Mechanical Engineers, Part C: Journal of Mechanical Engineering Science. 234 (2020) 2279-2293. https://doi.org/10.1177/0954406220904106.

[58] I. Sabree, J.E. Gough, B. Derby, Mechanical properties of porous ceramic scaffolds: Influence of internal dimensions, Ceramics International. $41 \quad$ (2015) 8425-8432. https://doi.org/10.1016/j.ceramint.2015.03.044.

[59] B. Basu, Mechanical Properties of Biomaterials, in: Biomaterials for Musculoskeletal Regeneration, Springer Singapore, Singapore, 2017: pp. 175-222. https://doi.org/10.1007/978-981-10-3059-8_6.

[60] P.N. Sudha, S. Kirubanandam, J. Av, V. N, R. Kannan, Corrosion of ceramic materials, in: Fundamental Biomaterials: Ceramics, 2018: pp. 223-250. https://doi.org/10.1016/B978-0-08102203-0.00009-3.

[61] M. Fulmer, I. Ison, C. Hankermayer, B. Constantz, J. Ross, Measurements of the Solubilities and Dissolution Rates of Several Hydroxyapatites, Biomaterials. 23 (2002) 751-5. https://doi.org/10.1016/S0142-9612(01)00180-6. 
[62] N. Eliaz, N. Metoki, Calcium Phosphate Bioceramics: A Review of Their History, Structure, Properties, Coating Technologies and Biomedical Applications, Materials. 10 (2017) 334. https://doi.org/10.3390/ma10040334.

[63] S. Vahabzadeh, M. Roy, A. Bandyopadhyay, S. Bose, Phase stability and biological property evaluation of plasma sprayed hydroxyapatite coatings for orthopedic and dental applications, Acta Biomater. 17 (2015) 47-55. https://doi.org/10.1016/j.actbio.2015.01.022.

[64] S.V. Dorozhkin, M. Epple, Biological and medical significance of calcium phosphates, Angew Chem Int Ed Engl. 41 (2002) 3130-3146. https://doi.org/10.1002/1521-3773(20020902)41:17<3130::AIDANIE3130>3.0.CO;2-1. 


\section{Supplementary material}

(a)

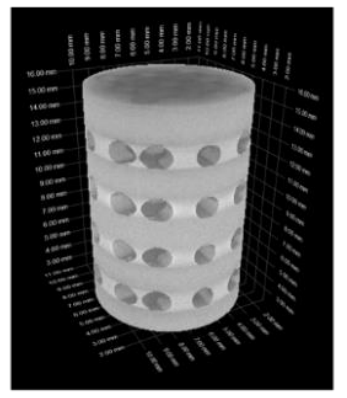

(d)

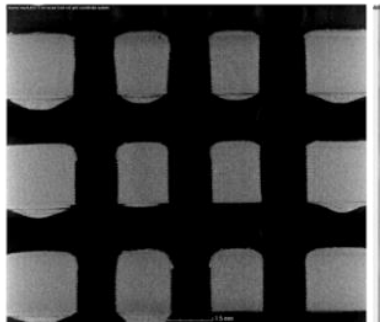

(b)

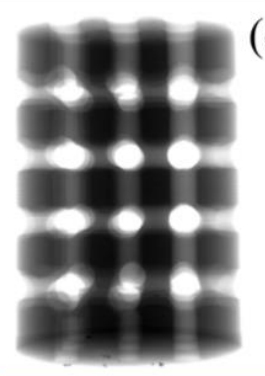

(c)
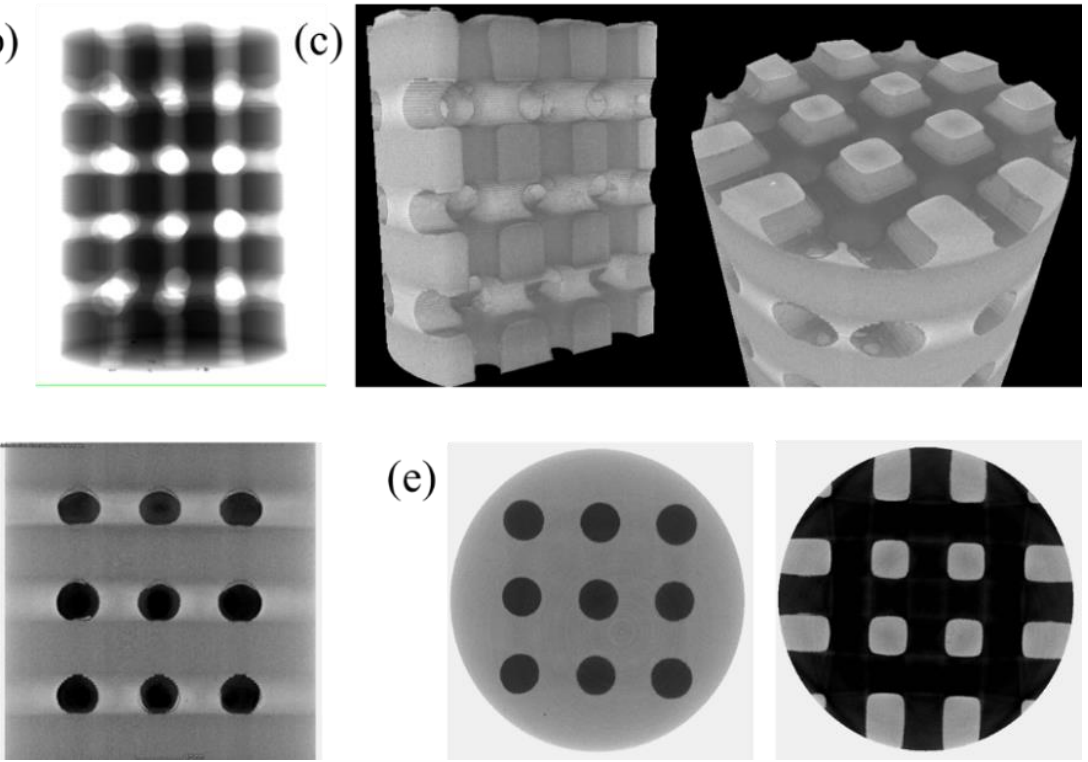

(e)

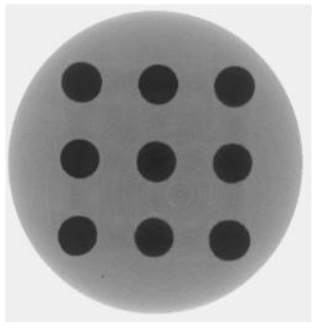

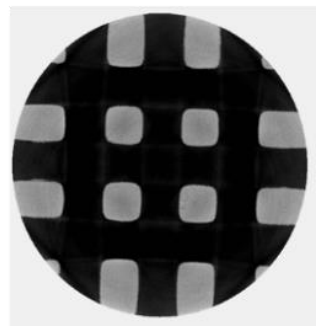

Figure S1. CT results for sintered HA scaffold printed at $0^{\circ}$ : (a) Volume reconstruction, (b) lateral view of the scaffold, $(c)$ volume cross-sectional views, (d) middle cross-sectional views (X, Z plane) of pores and struts, (e)middle cross-sectional views (X-Y plane) of pores and struts.

(a)

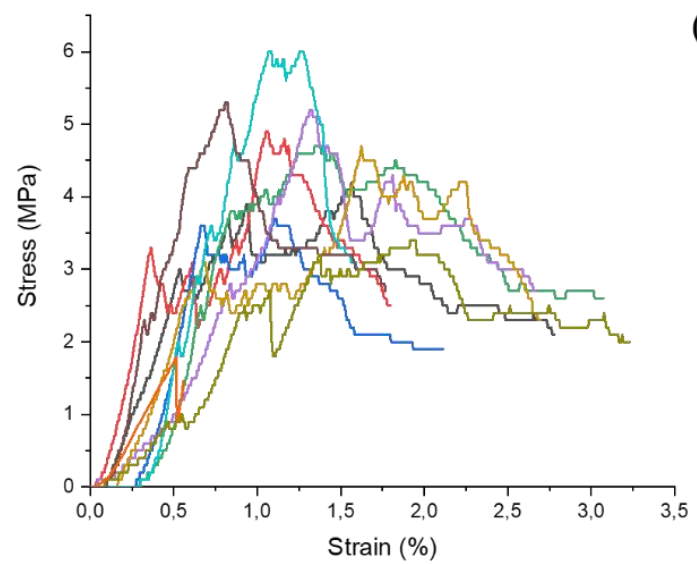

(b)

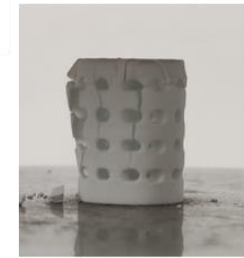

$45^{\circ}$

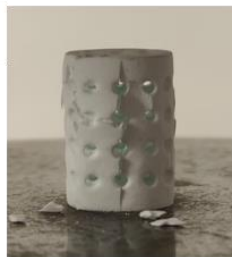

$90^{\circ}$

Figure S2. (a) Compilation of ten compressive stress-strain curves obtained for the sintered HA scaffolds at $45^{\circ}$ and (b) photographs of the specimens printed at different orientations $\left(0^{\circ}, 45^{\circ}\right.$, and $\left.90^{\circ}\right)$ after failure 